\title{
Molecular gas in nearby low-luminosity QSO host galaxies
}

\author{
T. Bertram ${ }^{1}$, A. Eckart ${ }^{1}$, S. Fischer ${ }^{1}$, J. Zuther $^{1}$, C. Straubmeier ${ }^{1}$, L. Wisotzki ${ }^{2}$, and M. Krips ${ }^{3}$ \\ 1 I. Physikalisches Institut, Universität zu Köln, Zülpicher Str. 77, 50937 Köln, Germany \\ e-mail: bertram@ph1.uni-koeln.de \\ 2 Astrophysikalisches Institut Potsdam, An der Sternwarte 16, 14482 Potsdam, Germany \\ ${ }^{3}$ Harvard-Smithsonian Center for Astrophysics, SMA project, 645 North A'ohoku Place, Hilo, HI 96720, USA
}

Received 31 March 2007 / Accepted 14 May 2007

\begin{abstract}
Aims. This paper addresses the global molecular gas properties of a representative sample of galaxies hosting low-luminosity quasistellar objects. An abundant supply of gas is necessary to fuel both the active galactic nucleus and any circum-nuclear starburst activity of QSOs. The connection between ultraluminous infrared galaxies and the host properties of QSOs is still subject to a controversial debate. Nearby low-luminosity QSOs are ideally suited to study the properties of their host galaxies because of their higher frequency of occurrence compared to high-luminosity QSOs in the same comoving volume and because of their small cosmological distance. Methods. We selected a sample of nearby low-luminosity QSO host galaxies that is free of infrared excess biases. All objects are drawn from the Hamburg-ESO survey for bright UV-excess QSOs, have $\delta>-30^{\circ}$ and redshifts that do not exceed $z=0.06$. The IRAM $30 \mathrm{~m}$ telescope was used to measure the ${ }^{12} \mathrm{CO}(1-0)$ and ${ }^{12} \mathrm{CO}(2-1)$ transition in parallel.

Results. 27 out of 39 galaxies in the sample have been detected. The molecular gas masses of the detected sources range from $0.4 \times 10^{9} M_{\odot}$ to $9.7 \times 10^{9} M_{\odot}$. The upper limits of the non-detected sources correspond to molecular gas masses between $0.3 \times 10^{9} M_{\odot}$ and $1.2 \times 10^{9} M_{\odot}$. We can confirm that the majority of galaxies hosting low-luminosity QSOs are rich in molecular gas. The properties of galaxies hosting brighter type I AGN and circumnuclear starformation regions differ from the properties of galaxies with fainter central regions. The overall supply of molecular gas and the spread of the line width distribution is larger. When comparing the farinfrared with the $\mathrm{CO}$ luminosities, the distribution can be separated into two different power-laws: one describing the lower activity Seyfert I population and the second describing the luminous QSO population. The separation in the $L_{\mathrm{FIR}} / L_{\mathrm{CO}}^{\prime}$ behavior may be explainable with differing degrees of compactness of the emission regions. We provide a simple model to describe the two power-laws. The sample studied in this paper is located in a transition region between the two populations.
\end{abstract}

Key words. galaxies: active - galaxies: ISM - quasars: general - radio lines: ISM - galaxies: Seyfert

\section{Introduction}

The investigation of the molecular gas content and its distribution in host galaxies of quasistellar objects (QSOs) is a key issue in the understanding of evolutionary sequences and environments of active galactic nuclei (AGN). All proposed sequences involve star formation and an abundant supply of material to fuel the starburst and the central engine.

Sanders et al. (1988) were the first to discuss a connection between ultraluminous infrared galaxies (ULIGs) and QSOs, suggesting that both represent different stages in an evolutionary sequence that starts with the collision of gas-rich spiral galaxies. In the course of the merger, circumnuclear starburst activity is triggered and a dominant part of available molecular gas is concentrated within the center of the merging galaxy. Several interferometric studies on individual ULIGs, like the archetypal ULIG Arp 220 (e.g. Scoville et al. 1991), reveal a massive concentration of molecular gas in the center ( $\$ 1 \mathrm{kpc}$ diameter). The bulk of $\mathrm{H}_{2}$ in these gas rich objects seems to be associated with this central gas accumulation. This massive concentration not only favors starburst activity but may also be required to drive the nuclear activity that becomes apparent in the QSO phase of the sequence. The more distant and more gas-rich submillimeter galaxies (SMGs) resemble scaled-up versions of the local ULIG population, also showing compact $\mathrm{CO}$ emission regions confined to the center. Tacconi et al. (2006) state a median diameter of $\leq 4 \mathrm{kpc}$ for 8 SMGs.
Contrary to the evolutionary model, Downes \& Solomon (1998) question the need for an AGN to power the FIR emission in ULIGs. They argue that extreme starbursts in circumnuclear molecular disks or rings are fully accountable for the high FIR luminosity. Following their line of arguments implies that, although several ULIGs with AGN are known, AGN are not mandatory for the ULIG appearance and ULIGs are not necessarily the predecessors of QSOs.

A recent study of Dunlop et al. (2003) on the morphological properties of QSO host galaxies discusses a decrease of the number density of disk dominated host galaxies ending in a complete depletion at $M_{\mathrm{V}} \leq-23$ (assuming $H_{0}=50 \mathrm{~km} \mathrm{~s}^{-1} \mathrm{Mpc}^{-1}$ ). From their analysis of Hubble space telescope and near infrared (NIR) data they conclude that QSO hosts have properties very similar to red, quiescent elliptical galaxies. These objects usually are gas depleted. Barthel (2006) argues for a more differentiated picture at least of intermediate luminosity QSO hosts and points to several indications of recent or ongoing star formation activity in these objecs.

Little is known about molecular gas in nearby QSO hosts: Evans et al. (2001, 2006) and Scoville et al. (2003) discuss small samples of QSO host galaxies selected from the PalomarGreen (PG) Bright Quasar Survey (Schmidt \& Green 1983). Few studies on individual sources add to the incomplete picture of molecular gas in local QSO hosts. A common denominator seems to be the presence of large amounts of molecular gas in 


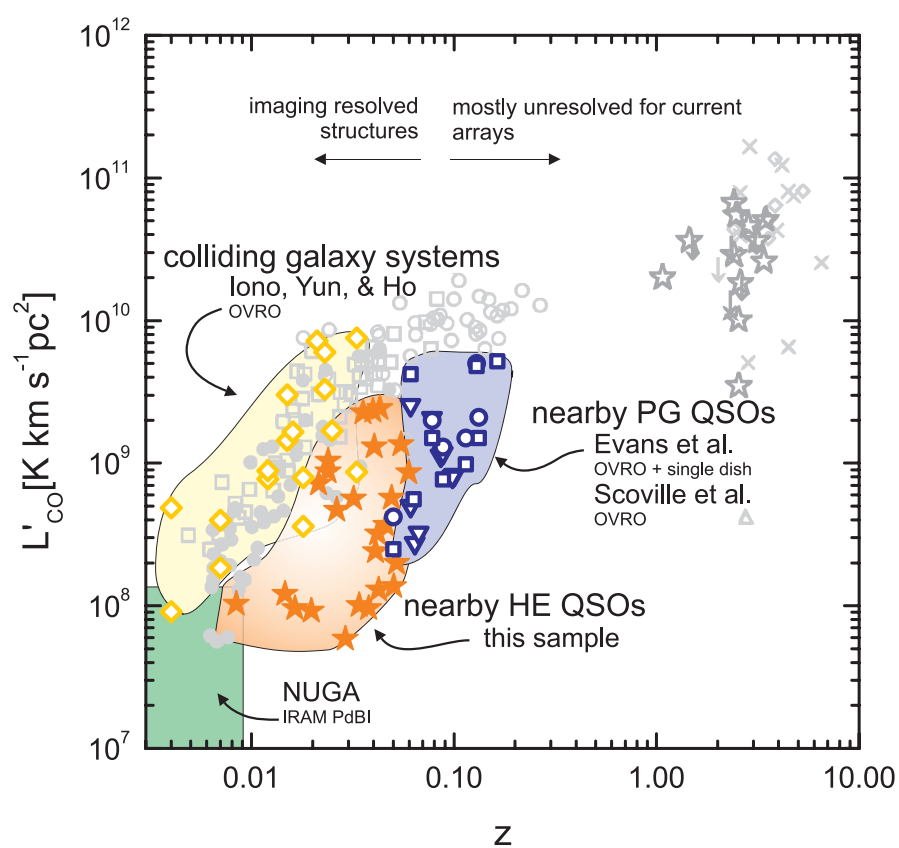

Fig. 1. $L_{\mathrm{CO}}-z$ distribution of already existing interferometric imaging studies of AGN hosts / interacting galaxies (thick outlined symbols) and the distribution of the nearby low-luminosity QSO sample. The underlying light gray symbols represent a compilation of the most $\mathrm{CO}$ luminous objects ((U)LIGs/SMGs/HzRGs/QSOs) measured so far (Greve et al. 2005) for the corresponding redshifts. The objects in the nearby low-luminosity QSO sample have redshifts that allow to resolve structures on the sub-kpc scale in the ${ }^{12} \mathrm{CO}(1-0)$ transition with current $\mathrm{mm}$ interferometers. (This figure is available in color in electronic form.)

the majority of these objects, which militates against a quiescent nature of host galaxies. The distribution of molecular gas, the extent and density of regions emitting $\mathrm{CO}$ line emission and similarities with or differences to local Seyfert I galaxies have not yet been studied on a solid statistical base. More detailed multiwavelength investigations of a larger sample of nearby QSOs are certainly beneficial in the controversial debate on the nature and history of QSOs.

Especially the separation of the starburst and the AGN component in extragalactic objects from the faint contribution of the underlying host galaxy require exceptionally high spatial resolution and sensitivity. The nearby QSOs with $z \leq 0.1$, therefore, represent an important link between the cosmologically local, less luminous AGN and the high redshift, high luminosity QSOs (at $z \geq 0.5$ ). Within this volume the accessibility of important structural information with state-of-the-art interferometers is feasible. In the millimeter domain, interferometers then provide sub-kpc resolutions that allow to probe the sizes of potentially compact circum-nuclear molecular gas reservoirs similar to the ULIG case (cf. Fig. 1). If the molecular gas is less confined to the center compared to the ULIG case it will be possible to study the distribution and potential signs of interaction on larger scales.

We have selected a sample of nearby UV excess QSOs or luminous Seyfert I galaxies, that are not only suitable for interferometric imaging in the millimeter wavelength domain but also allow for detailed imaging and spectroscopy in the NIR. For 41 members of the sample single-dish CO data was obtained (in one case also interferometric data). The sample is introduced in Sect. 2, together with a definition of the term "low-luminosity QSO" that is used throughout this paper. The results of the observations follow in Sect. 4. The data allows to draw conclusions on the total molecular gas content of low-luminosity QSOs and can be related to existing visible and far infrared quantities, as presented in Sect. 5. $H_{0}=75 \mathrm{~km} \mathrm{~s}^{-1} \mathrm{Mpc}^{-1}$ and $q_{0}=0.5$ are assumed throughout the paper.

\section{The nearby low-luminosity QSO sample}

The only selection criterion for the sample of nearby lowluminosity QSOs was their small cosmological distance: only objects with a redshift $z<0.060$ were chosen.

This redshift limit is based on a NIR spectroscopic constraint: it ensures the observability of the diagnostic $\mathrm{CO}(2-0)$ rotation vibrational band head absorption line, which is important for the stellar population analysis. This line is then still accessible in the $K$-band.

The members were selected entirely from an extended catalog of sources found in the Hamburg/ESO survey (HES). The HES (Wisotzki et al. 2000) is a wide angle survey for optically bright QSOs, with a well-defined flux limit of $B_{J} \lesssim 17.3$, varying from field to field, and a redshift coverage of $0<z<3.2$. QSO candidates were identified in digitized objective prism plate data by applying a color- and spectral feature based selection scheme. With the exception of objects listed in the Véron-Cetty \& Véron (1996) catalog, all candidates were subject to spectroscopic follow-up observations to confirm the object's identity and to reject false classifications.

The application of a starlike morphological criterion in many other QSO samples like the PG Bright Quasar Survey or the 10k catalog of the $2 \mathrm{dF}$ QSO Redshift Survey results in a significant degree of incompleteness at the low redshift end. One of the main advantages of the HES over other surveys is the consideration of extended objects. The HES sample, therefore, shows a high volume density of luminous type I AGN also at low cosmological distances. This circumstance makes the HES sample a valuable source of objects for a study of nearby QSOs. A total of 99 objects within the volume $0.01 \leq z \leq 0.06$ were identified by the HES. Thirty-nine of these form the subsample that is discussed in this paper. The objects in the subsample all have $\delta>-30^{\circ}$. To avoid potential selection biases, the composition of the subsample matches the full sample of nearby low-luminosity QSOs in terms of redshift distribution and percentage of IRAS detected sources.

It is important to note that no luminosity discrimination between QSOs and Seyfert I galaxies was applied by the HES - all luminous type I AGN showing broad emission lines ( $F W H M \geq$ $1000 \mathrm{~km} \mathrm{~s}^{-1}$ ) in their follow-up spectra were included in their catalog. This has a direct implication on the absolute brightness distribution of the sample here referred to as "nearby lowluminosity QSO sample". Our sample clearly probes the low luminosity tail of the local quasar luminosity function (Köhler et al. 1997). All objects in the sample have absolute $B_{\mathrm{J}}$ magnitudes exceeding (i.e., dimmer than) the traditional boundary ${ }^{1}$ $M_{\mathrm{B}} \sim-22$ between higher luminosity QSOs and lower luminosity Seyfert I galaxies. This boundary has no astrophysical motivation, as it was technologically induced at the time of its introduction (Schmidt \& Green 1983). However, to respect the commonly used definition of the term "QSO", we explicitely use the term "low-luminosity QSO" throughout the paper for objects identified in QSO surveys that may be fainter than the traditional boundary magnitude. Not only the HES but also the PG Bright Quasar Survey provide low-luminosity QSOs in their samples.

\footnotetext{
1 Translated to the cosmology used throughout the paper.
} 
Table 1. Journal of the observations carried out with the IRAM $30 \mathrm{~m}$ telescope in in September 2005, June 2006, and January 2007. Three previously detected sources are also listed. $z$ was taken from the HES. $t_{\text {int }}$ denotes the total on-source integration time for each receiver.

\begin{tabular}{|c|c|c|c|c|c|c|c|c|c|c|}
\hline \multirow[t]{2}{*}{$\overline{\text { Obj. }}$} & \multirow[t]{2}{*}{ Alt. name } & \multicolumn{3}{|c|}{$\overline{\mathrm{RA}(\mathrm{J} 2000)}$} & \multicolumn{3}{|c|}{$\overline{\overline{D e c}(\mathrm{~J} 2000)}$} & \multirow[t]{2}{*}{ 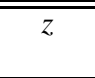 } & \multirow{2}{*}{$\begin{array}{c}\text { Obs. } \\
\text { run }\end{array}$} & \multirow{2}{*}{$\begin{array}{c}t_{\text {int }} \\
\text { [min] }\end{array}$} \\
\hline & & {$[\mathrm{h}$} & $\mathrm{m}$ & $\mathrm{s}]$ & {$\left[^{\circ}\right.$} & & "] & & & \\
\hline HE 0021-1810 & VCV2001 J002339.2-175355 & 00 & 23 & 39.4 & -17 & 53 & 53 & 0.053 & '07 & 118 \\
\hline HE 0021-1819 & NPM1G - 18.0010 & 00 & 23 & 55.3 & -18 & 02 & 50 & 0.052 & ’07 & 140 \\
\hline HE 0040-1105 & VIII Zw 36 & 00 & 42 & 36.8 & -10 & 49 & 21 & 0.041 & '06 & 112 \\
\hline HE $0045-2145$ & IRAS 00452-2145 & 00 & 47 & 41.3 & -21 & 29 & 27 & 0.021 & 05 & 45 \\
\hline HE 0108-4743 & IRAS 01089-4743 & 01 & 11 & 09.7 & -47 & 27 & 36 & $0.029^{1}$ & SEST & 76 \\
\hline HE 0114-0015 & SDSS J011703.58+000027.4 & 01 & 17 & 03.6 & +00 & 00 & 27 & 0.046 & 06 & 98 \\
\hline HE 0119-0118 & II Zw 1 & 01 & 21 & 59.8 & -01 & 02 & 25 & 0.054 & 05 & 60 \\
\hline HE 0150-0344 & IRAS 01505-0343 & 01 & 53 & 01.4 & -03 & 29 & 24 & 0.046 & '06 & 70 \\
\hline HE 0203-0031 & Mrk 1018 & 02 & 6 & 15.9 & -00 & 17 & 29 & 0.043 & 06 & 56 \\
\hline HE 0212-0059 & Mrk 590 & 02 & 14 & 33.6 & -00 & 46 & 00 & 0.027 & 05 & 60 \\
\hline HE 0224-2834 & AM 0224-283 & 02 & 26 & 25.7 & -28 & 20 & 59 & 0.060 & 06 & 55 \\
\hline HE 0227-0913 & Mrk 1044 & 02 & 30 & 05.4 & -08 & 59 & 53 & 0.017 & 06 & 46 \\
\hline HE 0232-0900 & NGC 985 & 02 & 34 & 37.7 & -08 & 47 & 16 & 0.043 & 06 & 32 \\
\hline HE 0253-1641 & NPM1G-16.0109 & 02 & 56 & 02.6 & -16 & 29 & 16 & 0.032 & 05 & 120 \\
\hline HE $0345+0056$ & IRAS $03450+0055$ & 03 & 47 & 40.2 & +01 & 05 & 14 & 0.029 & 05 & 117 \\
\hline HE $0351+0240$ & VCV2001 J035409.4+024931 & 03 & 54 & 09.4 & +02 & 49 & 30 & 0.034 & '06 & 74 \\
\hline HE 0412-0803 & MS 04124-0802 & 04 & 14 & 52.6 & -07 & 55 & 41 & 0.038 & ’06 & 115 \\
\hline HE 0429-0247 & RXS J04316-0241 & 04 & 31 & 37.0 & -02 & 41 & 25 & 0.041 & '06 & 61 \\
\hline HE 0433-1028 & Mrk 618 & 04 & 36 & 22.2 & -10 & 22 & 33 & 0.033 & 05 & 25 \\
\hline HE 0853-0126 & & 08 & 56 & 17.8 & -01 & 38 & 07 & 0.060 & '06 & 135 \\
\hline HE $0853+0102$ & & 08 & 55 & 54.3 & +00 & 51 & 10 & 0.052 & '06 & 120 \\
\hline HE 0934+0119 & Mrk 707 & 09 & 37 & 01.0 & +01 & 05 & 43 & 0.051 & '06 & 72 \\
\hline HE 0949-0122 & Mrk 1239 & 09 & 52 & 18.9 & -01 & 36 & 44 & 0.019 & 05 & 110 \\
\hline HE 1011-0403 & PG 1011-040 & 10 & 14 & 20.6 & -04 & 18 & 41 & 0.057 & '06 & 122 \\
\hline HE 1017-0305 & Mrk 1253 & 10 & 19 & 32.9 & -03 & 20 & 15 & 0.048 & 05 & 274 \\
\hline HE 1029-1831 & NPM1G-18.0348 & 10 & 31 & 57.3 & -18 & 46 & 34 & 0.040 & PdBI & \\
\hline HE 1107-0813 & & 11 & 09 & 48.5 & -08 & 30 & 15 & 0.057 & 07 & 80 \\
\hline HE $1108-2813$ & VCV2001 J111048.0-283004 & 11 & 10 & 48.0 & -28 & 30 & 03 & 0.023 & '05, '07 & 41 \\
\hline HE 1126-0407 & Mrk 1298, PG1126-041 & 11 & 29 & 16.6 & -04 & 24 & 08 & 0.060 & '05, ’07 & 41 \\
\hline HE 1237-0504 & NGC 4593 & 12 & 39 & 39.4 & -05 & 20 & 40 & 0.009 & 05 & 35 \\
\hline HE 1248-1356 & & 12 & 51 & 32.4 & -14 & 13 & 17 & 0.015 & '06 & 102 \\
\hline HE 1310-1051 & PG 1310-108 & 13 & 13 & 05.7 & -11 & 07 & 42 & 0.034 & ’07 & 64 \\
\hline HE 1330-1013 & & 13 & 32 & 39.1 & -10 & 28 & 53 & 0.022 & '07 & 59 \\
\hline HE 1338-1423 & & 13 & 41 & 12.9 & -14 & 38 & 40 & 0.041 & 07 & 85 \\
\hline HE 1353-1917 & VCV2001 J135636.7-193144 & 13 & 56 & 36.7 & -19 & 31 & 44 & 0.034 & '07 & 97 \\
\hline HE 1417-0909 & & 14 & 20 & 06.3 & -09 & 23 & 13 & 0.044 & 07 & 106 \\
\hline HE 2128-0221 & 6dF J2130499-020814 & 21 & 30 & 49.9 & -02 & 08 & 15 & 0.052 & '05, ’07 & 295 \\
\hline HE 2211-3903 & & 22 & 14 & 42.0 & -38 & 48 & 24 & 0.039 & SEST & 114 \\
\hline HE 2222-0026 & SDSS J222435.29-001103.8 & 22 & 24 & 35.3 & -00 & 11 & 04 & 0.058 & '07 & 225 \\
\hline HE 2233-0124 & VCV2001 J223541.9+013933 & 22 & 35 & 41.9 & -01 & 39 & 33 & 0.056 & 07 & 224 \\
\hline HE 2302-0857 & Mrk 926 & 23 & 04 & 43.4 & -08 & 41 & 09 & 0.046 & '07 & 150 \\
\hline
\end{tabular}

${ }^{1}$ The redshift for HE 0108-4743 was taken from Véron-Cetty \& Véron (1996) and not spectroscopically verified by the HES. Since it differs significantly from other visual redshift data (Lawrence et al. 1999), the value was excluded from the redshift discussion in Sect. 5.2.

The restriction to type I AGN in the HES naturally resulted in the exclusion of type II AGN in the nearby low-luminosity QSO sample. For this reason the comparison with literature data in Sect. 5 was also restricted to type I AGN.

Several members of the nearby low-luminosity QSO sample were already subject to studies in the NIR and mm wavelength domain. First NIR imaging and spectroscopic results of nearby low-luminosity QSO sample members are presented in Fischer et al. (2006). A series of observations carried out with SEST and BIMA (Bertram et al. 2006) preceded the observations presented in this paper. In these first runs a slightly differently composed sample was scanned for millimetric $\mathrm{CO}$ emission. The primary goal was to identify the $\mathrm{CO}$ brightest objects for high resolution interferometric follow-up observations. The resulting SEST spectra and CO properties of two detected nearby HES objects, HE 0108-4743 and HE 2211-3903, are included in Sects. 4 and 5 . However, due to the higher detection limit, they were not included in the FIR unbiased subsample discussed in Sect. 5.1. The third detection, HE 1029-1831, was included. Follow-up observations with the Plateau de Bure Interferometer allowed to resolve the central region in this exemplary case. A detailed analysis can be found in Krips et al. (2007).

\section{Observations and data reduction}

In this paper we report on sensitive observations carried out with the IRAM 30 m telescope on Pico Veleta (Spain) in September 2005, June 2006 and January 2007.

Twenty-six out of 38 observed sources were detected (cf. Table 1). In most cases the detection limits were much lower than in the preceding observing runs. However, part of the data obtained in June 2006 suffered from mediocre weather conditions and had to be excluded from further analysis. Moreover, in several cases the observing frequencies had to be based only on the redshifts provided by the HES. Although nominally uncertain by $300 \mathrm{~km} \mathrm{~s}^{-1}$, in some cases the Hamburg/ESO based 
systemic velocities deviated from the measured systemic velocity centroids by up to $750 \mathrm{~km} \mathrm{~s}^{-1}$ (cf. Sect. 5.2). A certain fraction of weak sources may not have been detected because of this de facto uncertainty.

The $\mathrm{A}$ and $\mathrm{B}$ receivers were used together with the $1 \mathrm{MHz}$ and $4 \mathrm{MHz}$ filter banks to acquire ${ }^{12} \mathrm{CO}(1-0)$ and ${ }^{12} \mathrm{CO}(2-1)$ emission line data in parallel. The configuration of the backends allowed for a velocity coverage $\Delta v$ between $1300 \mathrm{~km} \mathrm{~s}^{-1}$ and $1400 \mathrm{~km} \mathrm{~s}^{-1}$ at the redshifted CO lines. The compactness of the sources allowed to take advantage of the wobbling secondary and the resulting excellent baselines. In the three observing runs useful data with a total of $\sim 65 \mathrm{~h}$ on-source integration time was obtained.

The data reduction was carried out using the IRAM software CLASS/GILDAS. Each polarization direction was analyzed individually and both directions were averaged whenever appropriate. In all cases it was sufficient to subtract linear baselines. To increase the signal-to-noise ratio, most of the spectra (cf. Fig. 2) were hanning smoothed. In order to obtain main beam temperatures, the main beam efficiency $B_{\text {eff }}=0.75$ and the forward efficiency $F_{\text {eff }}=0.95$ were applied to $T_{\mathrm{A}}^{*}$ at $\sim 110 \mathrm{GHz}$ and $B_{\text {eff }}=0.54$ and $F_{\text {eff }}=0.91$ to $T_{\mathrm{A}}^{*}$ at $\sim 220 \mathrm{GHz}$. The line intensities $I_{\mathrm{CO}}$ represent main beam temperatures integrated over the full velocity range of the emission line. The errors $\Delta I_{\mathrm{CO}}$ were determined by the geometric average of the line error $\Delta I_{\mathrm{L}}=\sigma v_{\text {res }} \sqrt{N_{\mathrm{L}}}$ and the baseline error $\Delta I_{\mathrm{B}}=\sigma v_{\text {res }} N_{\mathrm{L}} / \sqrt{N_{\mathrm{B}}}$, where $\sigma$ is the channel-to-channel rms noise, $v_{\text {res }}$ the spectral resolution, $N_{\mathrm{L}}$ the number of channels over which the line is distributed and $N_{\mathrm{B}}$ the number of channels used for the baseline fit.

\section{Results}

The CO line luminosity $L_{\mathrm{CO}}^{\prime}$ of the ${ }^{12} \mathrm{CO}(1-0)$ transition can be expressed as (Solomon et al. 1992a):

$L_{\mathrm{CO}}^{\prime}=23.5 \Omega_{\mathrm{S} \star B} D_{L}^{2} I_{\mathrm{CO}}(1+z)^{-3}\left[\mathrm{~K} \mathrm{~km} \mathrm{~s}^{-1} \mathrm{pc}^{2}\right]$,

i.e., as a function of the velocity-integrated line intensity $I_{\mathrm{CO}}$ in units of $\mathrm{K} \mathrm{km} \mathrm{s}^{-1}$, the luminosity distance $D_{\mathrm{L}}$ measured in $\mathrm{Mpc}$, and the solid angle of the source convolved with the telescope beam $\Omega_{\mathrm{S} \star \mathrm{B}}$ in square arcseconds. For sources with solid angles much smaller than the telescope beam, $\Omega_{\mathrm{S} \star \mathrm{B}}$ can be approxi-

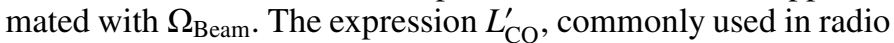
astronomy, and the general expression of the line luminosity $L_{\mathrm{CO}}$ are related by $L_{\mathrm{CO}}=\left(8 \pi k v_{\text {rest }}^{3} / c^{3}\right) L_{\mathrm{CO}}^{\prime}$ (Solomon et al. 1992b). $1 \mathrm{~K} \mathrm{~km} \mathrm{~s}^{-1}$ corresponds to $\sim 1.9 \times 10^{29} \mathrm{erg} \mathrm{s}^{-1}$ at the $115 \mathrm{GHz}$ transition.

Established correlations (Young et al. 1995) between the $25.0 B_{\mathrm{mag}} \operatorname{arcsec}^{-2}$ isophotal diameter $D_{25}$ of galaxies and the size of their $\mathrm{CO}$ emission region were used to confirm that, with the exception of HE 0212-0059 and HE 0232-0900, the sources should all be smaller than the $\sim 22^{\prime \prime}$ telescope beam resulting at $\sim 115 \mathrm{GHz}$. For the two exceptional cases it is likely that the overall $\mathrm{CO}$ line luminosity is underestimated in the present study. In the case of HE 0232-0900, already published data (Horellou et al. 1995, see below) allowed to determine a factor $\sim 1.7$ by which the current data is too low. Because of the very similar optical extent, a similar factor can be assumed for HE 0212-0059.

The integrated line intensity, the systemic velocity (represented by the flux-weighted centroid of the $\mathrm{CO}(1-0)$ line), the linewidth (FWZI), the resolution of the smoothed spectrum as well as the derived CO luminosity of each detected source is shown in Table 2. Also shown are estimates for the molecular gas masses. They were obtained by applying a $M\left(\mathrm{H}_{2}\right) / L_{\mathrm{CO}}^{\prime}$ conversion factor $\alpha$ to the $\mathrm{CO}$ luminosity. The determination of the $\mathrm{H}_{2}$ content using $L_{\mathrm{CO}}^{\prime}$ as tracer has to be handled with care. Commonly used is a value of $\alpha=4.8 M_{\odot}\left(\mathrm{K} \mathrm{km} \mathrm{s}^{-1} \mathrm{pc}^{2}\right)^{-1}$ (Solomon \& Barrett 1991), derived from galactic molecular cloud observations. The common approach of using a 'standard' conversion factor derived from galactic observations has the shortcoming of disregarding the dependency of $\alpha$ on the metalicity of the region of interest (Israel 1997). For a sample of ultraluminous infrared galaxies Downes \& Solomon (1998) state a conversion factor that is 5 times lower. Within this paper $\alpha=4 M_{\odot}\left(\mathrm{K} \mathrm{km} \mathrm{s}^{-1} \mathrm{pc}^{2}\right)^{-1}$ is adopted to allow for direct comparison with the results of Evans et al. $(2001,2006)$ or Scoville et al. (2003).

A subset of the sources had been subject to molecular gas studies before. The published data confirms the results listed in Table 2. The ${ }^{12} \mathrm{CO}(1-0)$ emission of the extended source HE 0232-0900 was mapped with the IRAM 30 m telescope by Horellou et al. (1995). The value for $I_{\mathrm{CO}}$ of the central position agrees within $18 \%$. After correction for the different beam sizes, the ${ }^{12} \mathrm{CO}(1-0)$ detections of HE 0227-0913 with the NRO 45 m telescope by Vila-Vilaró et al. (1998), of HE 1237-0504 with SEST by Curran et al. (2000), and of HE 0433-1028 with SEST by Strong et al. (2004) agree within $10 \%$ with the values presented in this paper. HE 1237-0504 was also observed by Maiolino et al. (1997) with the NRAO $12 \mathrm{~m}$ telescope. Their beam-size corrected values for this object, for HE 0212-0059, and for HE 0949-0122, however, exceed the values presented here by a factor 2-3. For HE 0212-0059, the difference may be explained with the extension of the galaxy and the different beam sizes. The other results, however, are in contrast with the good agreements of the 4 other independent measurements mentioned above, which provide a high level of confidence in the data presented here.

\section{Discussion}

\subsection{Detection rate and molecular gas mass}

About $60 \%$ of the mostly southern sources in the nearby lowluminosity QSO sample have a declination $\geq-30^{\circ}$ and can be observed with the IRAM 30 m telescope. Of these, about $65 \%$ have been subject to our study. They show a redshift distribution similar to the full nearby low-luminosity QSO sample (Fig. 3). Within the restricted redshift range considered in this sample, the number of objects per redshift bin is purely volume dependent the number increases with increasing $z$.

When plotted against redshift (Fig. 4), the particular position of the sample in between previously studied local Seyfert I and more luminous QSO host galaxies becomes apparent. The omission of a far infrared (FIR) selection and the on average larger distance compared to the local Seyfert I population resulted in the inclusion of several objects with FIR flux densities below the IRAS detection limit (cf. Sect. 5.4). In several of these cases we still were able to detect $\mathrm{CO}$ emission.

Our $3 \sigma$ detection limits agree with the limits in the PG QSO host studies by Evans et al. $(2001,2006)$ and Scoville et al. (2003), carried out with the OVRO array and the IRAM $30 \mathrm{~m}$ telescope. Different from Evans et al. (2001, 2006), the volume limited sample of Scoville et al. (2003), consisting of $12 \mathrm{ob}-$ jects, is not confined to FIR selected sources. Therefore, a direct comparison with their results is appropriate. At redshifts exceeding 0.06 , the common detection limit prevents the detection of 

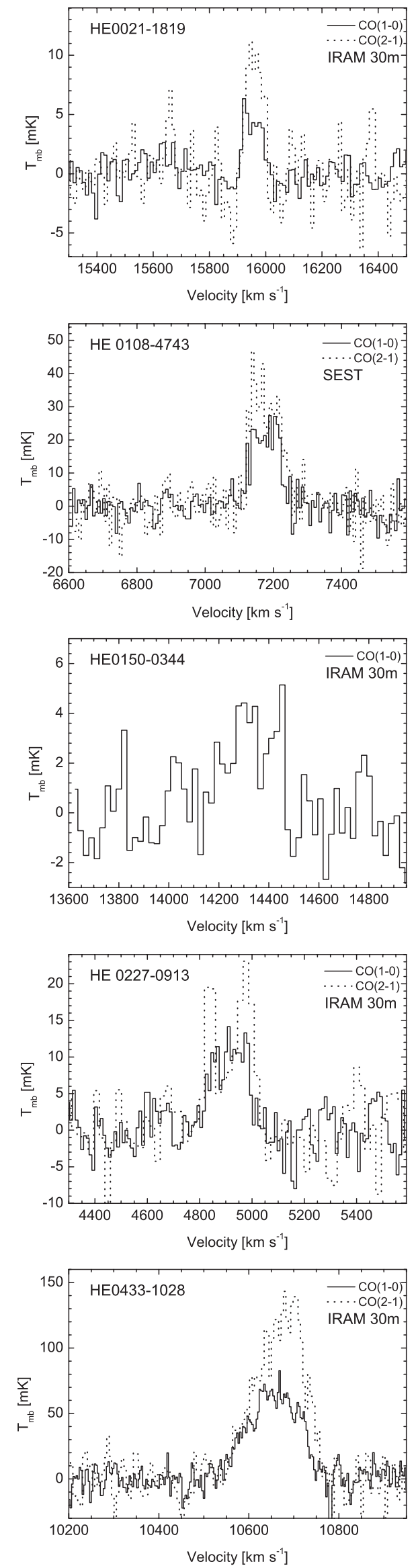
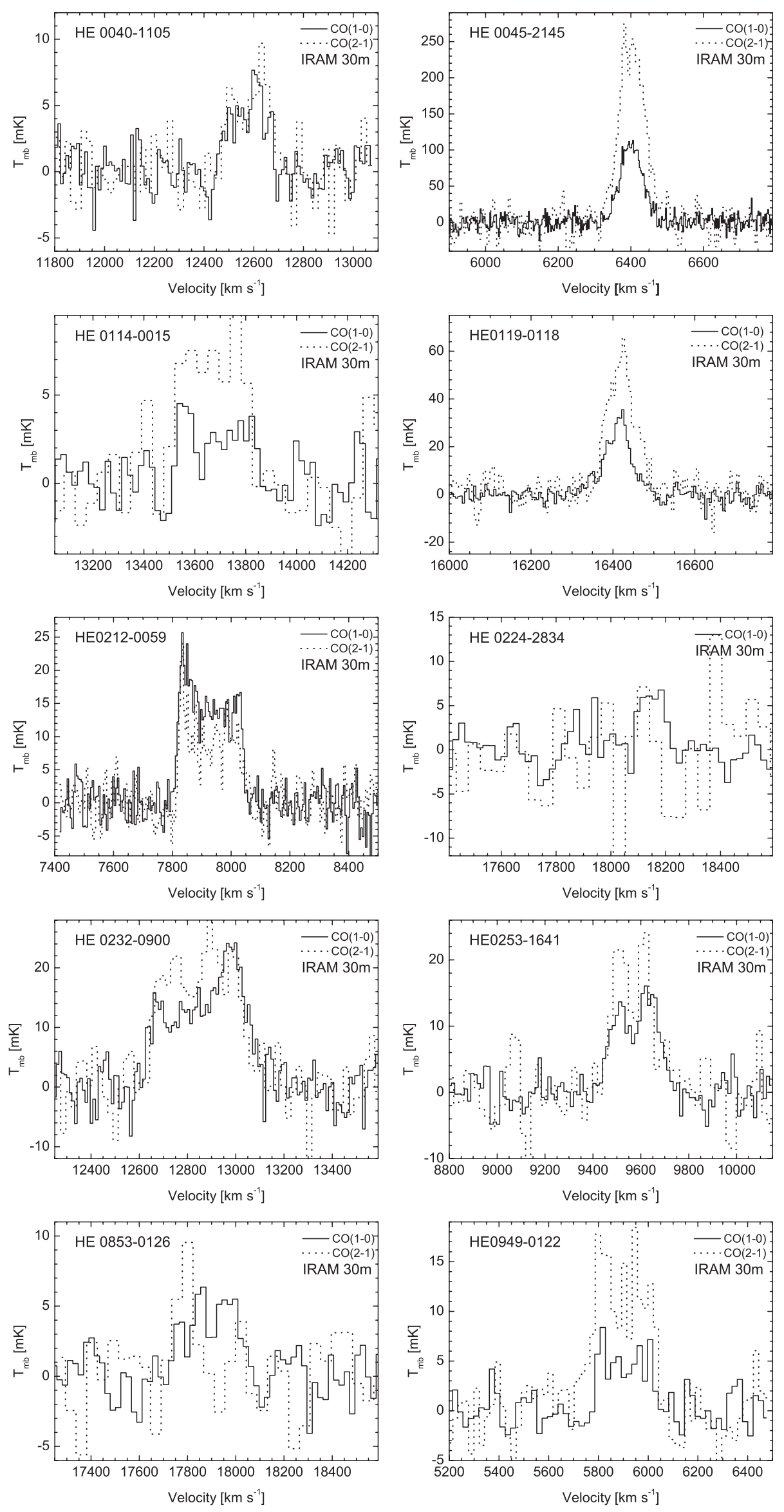

Fig. 2. ${ }^{12} \mathrm{CO}$ spectra of detected host galaxies. In two cases, the weather conditions prevented reliable measurements of the ${ }^{12} \mathrm{CO}(2-1)$ transition. 

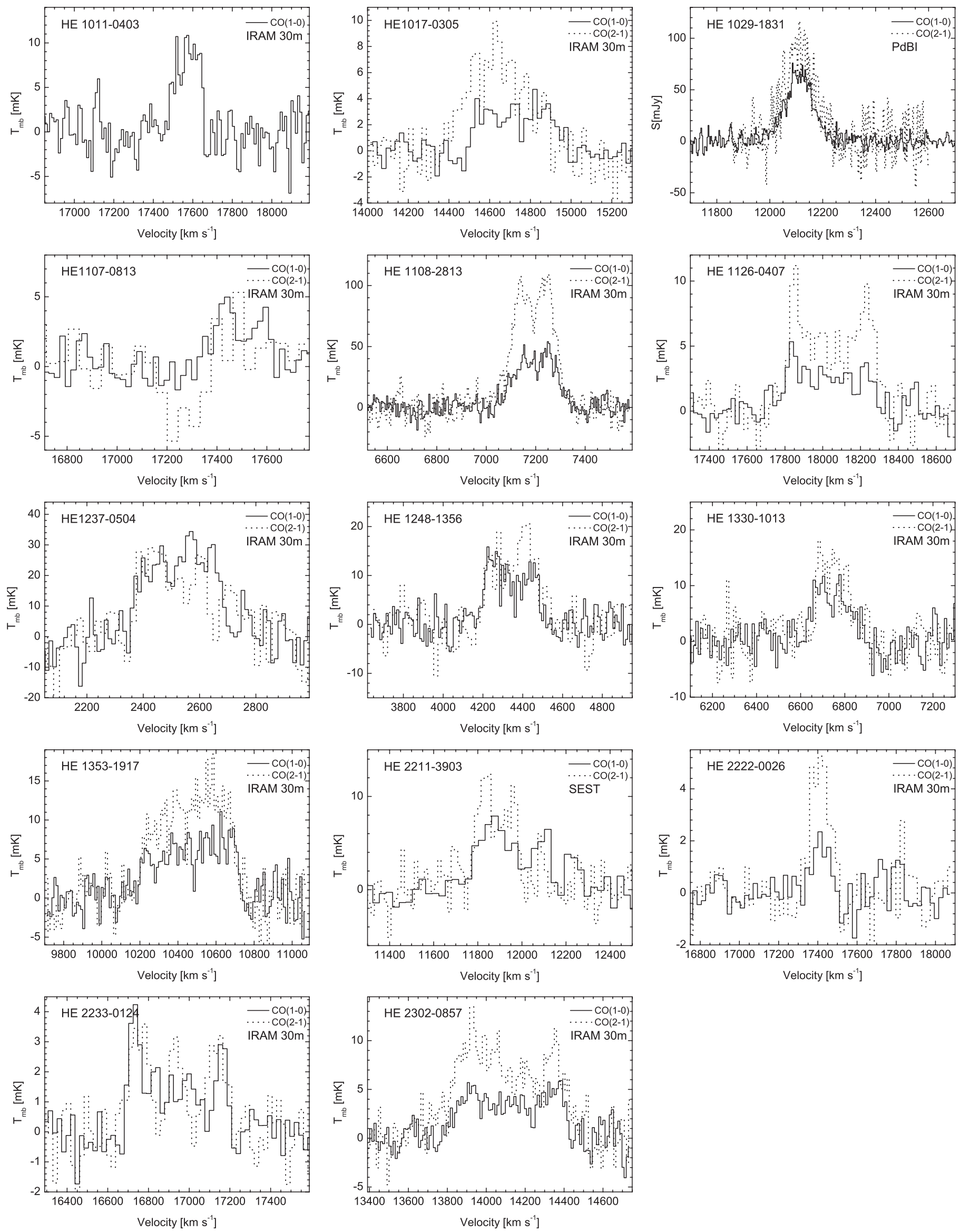

Fig. 2. continued. 
Table 2. Summary of ${ }^{12} \mathrm{CO}$ properties. Unless otherwise noted, the data was acquired with the IRAM $30 \mathrm{~m}$ telescope.

\begin{tabular}{|c|c|c|c|c|c|c|c|c|c|c|c|}
\hline \multirow[b]{2}{*}{ Obj. } & \multirow[b]{2}{*}{$\begin{array}{l}v_{0}(\mathrm{LSR}) \\
{\left[\mathrm{km} \mathrm{s}^{-1}\right]}\end{array}$} & \multirow[b]{2}{*}{$\begin{array}{c}D_{\mathrm{L}} \\
{[\mathrm{Mpc}]}\end{array}$} & \multicolumn{6}{|c|}{$\bar{~}{ }^{12} \mathrm{CO}(1-0)$} & \multicolumn{3}{|c|}{${ }^{{ }^{12} \mathrm{CO}(2-1)}$} \\
\hline & & & $\begin{array}{c}I_{\mathrm{CO}}{ }^{1} \\
{\left[\mathrm{~K} \mathrm{~km} \mathrm{~s}^{-1}\right]}\end{array}$ & $\begin{array}{c}\Delta v_{\text {FWZI }} \\
{\left[\mathrm{km} \mathrm{s}^{-1}\right]}\end{array}$ & $\begin{array}{c}v_{\text {res }} \\
{\left[\mathrm{km} \mathrm{s}^{-1}\right]}\end{array}$ & $L S^{2}$ & $\begin{array}{c}L_{\mathrm{CO}}^{\prime} / 10^{8} 3 \\
{\left[\mathrm{~K} \mathrm{~km} \mathrm{~s}^{-1} \mathrm{pc}^{2}\right]}\end{array}$ & $\begin{array}{c}M\left(\mathrm{H}_{2}\right) \\
{\left[10^{9} M_{\odot}\right]}\end{array}$ & $\begin{array}{c}I_{\mathrm{CO}}{ }^{1} \\
{\left[\mathrm{~K} \mathrm{~km} \mathrm{~s}^{-1}\right]}\end{array}$ & $\begin{array}{c}\Delta v_{\text {FWZI }} \\
{\left[\mathrm{km} \mathrm{s}^{-1}\right]}\end{array}$ & $\begin{array}{c}v_{\text {res }} \\
{\left[\mathrm{km} \mathrm{s}^{-1}\right]}\end{array}$ \\
\hline HE 0021-1819 & 15954 & 215.5 & $0.39 \pm 0.04$ & 110 & 11.0 & $\wedge$ & 2.1 & 0.8 & $0.68 \pm 0.1$ & 121 & 11.0 \\
\hline HE 0040-1105 & 12578 & 169.4 & $0.96 \pm 0.08$ & 238 & 10.8 & $\Pi$ & 3.2 & 1.3 & $1.19 \pm 0.15$ & 238 & 22.0 \\
\hline HE $0045-2145$ & 6403 & 85.8 & $8.20 \pm 0.18$ & 133 & 9.0 & $\wedge$ & 7.2 & 2.9 & $22.39 \pm 0.59$ & 181 & 5.3 \\
\hline HE $0108-4743^{4}$ & 7175 & 96.2 & $2.24 \pm 0.07$ & 118 & 7.4 & $\sqcap$ & 10.4 & 4.2 & $3.67 \pm 0.1$ & 160 & 7.4 \\
\hline HE 0114-0015 & 13682 & 184.5 & $0.92 \pm 0.13$ & 326 & 21.8 & $\Pi$ & 3.6 & 1.5 & $2.24 \pm 0.38$ & 305 & 43.5 \\
\hline HE 0119-0118 & 16412 & 221.7 & $2.38 \pm 0.09$ & 165 & 5.5 & $\wedge$ & 13.5 & 5.4 & $4.70 \pm 0.17$ & 143 & 5.5 \\
\hline HE 0150-0344 & 14329 & 193.3 & $0.89 \pm 0.15$ & 305 & 21.8 & $\sqcap$ & 3.8 & 1.5 & & & \\
\hline HE 0212-0059 & 7921 & 106.3 & $3.51 \pm 0.11$ & 240 & 5.3 & $\sqcap$ & $4.7^{8}$ & $1.9^{8}$ & $2.42 \pm 0.12$ & 251 & 5.3 \\
\hline HE 0224-2834 & 18150 & 245.6 & $0.71 \pm 0.11^{5}$ & 132 & 21.1 & $\wedge$ & 4.9 & 2.0 & $<0.46$ & 132 & 22.1 \\
\hline HE $0227-0913$ & 4914 & 65.8 & $1.86 \pm 0.17$ & 254 & 10.6 & $\sqcap$ & 1.0 & 0.4 & $2.96 \pm 0.31$ & 254 & 21.1 \\
\hline HE 0232-0900 & 12886 & 173.6 & $6.92 \pm 0.34$ & 597 & 10.9 & $\sqcap$ & $24.3^{9}$ & $9.7^{9}$ & $8.13 \pm 0.66$ & 586 & 22.0 \\
\hline HE 0253-1641 & 9580 & 128.7 & $2.89 \pm 0.15$ & 282 & 13.4 & $\sqcap$ & 5.6 & 2.3 & $3.89 \pm 0.37$ & 301 & 21.0 \\
\hline HE 0433-1028 & 10651 & 143.3 & $9.42 \pm 0.27$ & 252 & 3.4 & $\wedge$ & 22.6 & 9.0 & $16.55 \pm 0.48$ & 199 & 5.4 \\
\hline HE 0853-0126 & 17899 & 242.1 & $1.28 \pm 0.15$ & 331 & 22.1 & $\sqcap$ & 8.6 & 3.4 & $0.82 \pm 0.36$ & 309 & 44.1 \\
\hline HE 0949-0122 & 5905 & 79.1 & $1.23 \pm 0.14$ & 276 & 21.2 & $\Pi$ & 0.9 & 0.4 & $3.55 \pm 0.24$ & 318 & 21.2 \\
\hline HE 1011-0403 & 17572 & 237.6 & $1.37 \pm 0.12$ & 187 & 11.0 & $\sqcap$ & 8.9 & 3.5 & 7 & & \\
\hline HE 1017-0305 & 14737 & 198.9 & $1.25 \pm 0.10$ & 458 & 21.8 & $\Pi$ & 5.7 & 2.3 & $2.44 \pm 0.23$ & 458 & 21.8 \\
\hline HE $1029-1831^{6}$ & 12112 & 163.1 & $4.24 \pm 0.2$ & 254 & 3.4 & $\wedge$ & 13.1 & 5.3 & $5.05 \pm 0.2$ & 254 & 3.4 \\
\hline HE 1107-0813 & 17481 & 236.4 & $0.82 \pm 0.10$ & 308 & 22.0 & $\sqcap$ & 5.2 & 2.1 & $<0.3$ & 310 & 44.0 \\
\hline HE $1108-2813$ & 7198 & 96.5 & $8.32 \pm 0.28$ & 309 & 5.3 & $\sqcap$ & 9.2 & 3.7 & $18.84 \pm 0.47$ & 320 & 5.3 \\
\hline HE 1126-0407 & 18006 & 243.6 & $1.52 \pm 0.15$ & 640 & 22.1 & $\sqcap$ & 10.3 & 4.1 & $3.02 \pm 0.20$ & 551 & 22.1 \\
\hline HE 1237-0504 & 2531 & 33.8 & $7.52 \pm 0.57$ & 302 & 13.1 & $\sqcap$ & 1.0 & 0.4 & $6.04 \pm 1.01$ & 315 & 21.0 \\
\hline HE $1248-1356$ & 4338 & 58.0 & $2.96 \pm 0.19$ & 348 & 10.5 & $\sqcap$ & 1.2 & 0.5 & $4.23 \pm 0.39$ & 338 & 21.1 \\
\hline HE $1330-1013$ & 6744 & 90.4 & $1.82 \pm 0.16$ & 266 & 10.6 & $\Pi$ & 1.8 & 0.7 & $2.4 \pm 0.2$ & 245 & 10.6 \\
\hline HE 1353-1917 & 10472 & 140.8 & $3.25 \pm 0.22$ & 592 & 10.7 & $\Pi$ & 7.6 & 3.0 & $5.5 \pm 0.3$ & 549 & 10.8 \\
\hline HE 2211-3903 & 11945 & 160.8 & $1.87 \pm 0.11$ & 466 & 30.0 & $\sqcap$ & 23.2 & 9.3 & $1.85 \pm 0.06$ & 260 & 15.1 \\
\hline HE 2222-0026 & 17414 & 235.5 & $0.21 \pm 0.04$ & 154 & 22.0 & $\wedge$ & 1.3 & 0.5 & $0.61 \pm 0.06$ & 176 & 22.0 \\
\hline HE $2233+0124$ & 16913 & 228.6 & $0.89 \pm 0.08$ & 506 & 22.0 & $\sqcap$ & 5.4 & 2.1 & $0.95 \pm 0.12$ & 506 & 22.0 \\
\hline HE 2302-0857 & 14120 & 190.4 & $2.31 \pm 0.16$ & 653 & 10.9 & $\sqcap$ & 9.7 & 3.9 & $5.17 \pm 0.29$ & 817 & 10.9 \\
\hline \multicolumn{12}{|c|}{ Non-detections } \\
\hline HE 0021-1810 & 16039 & 216.6 & $<0.07$ & 315 & 43.8 & & $<1.1$ & $<0.5$ & $<0.12$ & & 43.8 \\
\hline HE 0203-0031 & 12723 & 171.4 & $<0.16$ & 315 & 43.4 & & $<1.6$ & $<0.6$ & $<0.51$ & & 43.4 \\
\hline HE $0345+0056$ & 8994 & 120.8 & $<0.15$ & 315 & 42.9 & & $<0.8$ & $<0.3$ & $<0.26$ & & 42.9 \\
\hline HE $0351+0240$ & 10793 & 137.0 & $<0.19$ & 315 & 43.1 & & $<1.2$ & $<0.5$ & 7 & & \\
\hline HE 0412-0803 & 11392 & 152.9 & $<0.14$ & 315 & 43.2 & & $<1.2$ & $<0.5$ & 7 & & \\
\hline HE 0429-0247 & 12441 & 165.5 & $<0.31$ & 315 & 43.4 & & $<2.9$ & $<1.2$ & 7 & & \\
\hline HE $0853+0102$ & 15589 & 210.5 & $<0.16$ & 315 & 43.8 & & $<2.4$ & $<1.0$ & 7 & & \\
\hline HE $0934+0119$ & 15091 & 203.7 & $<0.12$ & 315 & 43.7 & & $<1.7$ & $<0.7$ & $<0.24$ & & 43.7 \\
\hline HE 1310-1051 & 10193 & 137.0 & $<0.16$ & 315 & 43.0 & & $<1.0$ & $<0.4$ & $<0.23$ & & 43.0 \\
\hline HE $1338-1423$ & 12528 & 168.8 & $<0.15$ & 315 & 43.4 & & $<1.5$ & $<0.6$ & $<0.21$ & & 43.4 \\
\hline HE 1417-0909 & 13191 & 177.8 & $<0.09$ & 315 & 43.4 & & $<1.0$ & $<0.4$ & $<0.18$ & & 43.4 \\
\hline HE 2128-0221 & 15828 & 213.8 & $<0.08$ & 315 & 43.8 & & $<1.3$ & $<0.5$ & $<0.12$ & & 43.8 \\
\hline
\end{tabular}

${ }^{1}$ Upper limits and errors represent $1 \sigma .{ }^{2}$ Line shape; $\wedge$ denotes a triangular shaped line profile, as it is expected for turbulent line-of-sight velocity dispersion; $\sqcap$ represents boxy or double-horn line profiles indicative for emission from an inclined, rotating disk. ${ }^{3}$ For the upper $L_{\mathrm{CO}}^{\prime}$ limits, the average linewidth $\Delta v=315 \mathrm{~km} \mathrm{~s}^{-1}$ and $3 \sigma$ detection limits were considered. ${ }^{4}$ SEST $15 \mathrm{~m}$ telescope measurement. A beam efficiency $B_{\text {eff }}=0.7$ was applied to $T_{\mathrm{A}}^{*}$ and a $45^{\prime \prime}$ beam was considered for $L_{\mathrm{CO}}^{\prime} \cdot{ }^{5} 3 \sigma$ detection level for $T_{\mathrm{mb}}{ }^{6}$ IRAM PdBI measurement (Krips et al. 2007), resulting in $S \Delta v=(21 \pm 1) \mathrm{Jy} \mathrm{km} \mathrm{s}^{-1}$ for ${ }^{12} \mathrm{CO}(1-0)$ and $S \Delta v=(25 \pm 1) \mathrm{Jy} \mathrm{km} \mathrm{s}{ }^{-1}$ for ${ }^{12} \mathrm{CO}(2-1) . S / T_{\mathrm{mb}}=4.95 \mathrm{Jy} / \mathrm{K}$ was used to compare the PdBI results with the $30 \mathrm{~m}$ telescope data. ${ }^{7} 1 \mathrm{~mm}$ data not reliable due to high water vapor. ${ }^{8}$ Source is likely to be extended. Value probably too low by an estimated factor $1.7 .{ }^{9}$ Source is not compact. Values too low by a factor $\sim 1.7$

galaxies with a molecular gas content of below $10^{9} M_{\odot}-$ only gas rich objects are then detectable. The redshifts of virtually all PG QSOs exceed this value. Nevertheless, sample of Scoville et al. (2003) resulted in a detection rate of $75 \%$ which lets the authors conclude that the majority of luminous, low-redshift QSOs have gas-rich host galaxies. This picture may become more complicated when considering the incompleteness of the PG survey at low redshifts. The rejection of extended objects may cause an underrepresentation of bulge-dominated galaxies with a lower gas content. On the other hand, due to the arbitrariness of the $M_{\mathrm{B}} \sim-22$ borderline, low-luminosity QSOs above the line still may have host properties that are similar to their brighter equivalent. With $70 \%$ the detection rate of the IRAM 30 m observations presented here is almost identical to the rate of Scoville et al. (2003). The molecular gas masses of the detected host galaxies range from $0.4 \times 10^{9} M_{\odot}$ to $9.7 \times 10^{9} M_{\odot}$ with an average molecular gas mass of $2.8 \times 10^{9} M_{\odot}\left(3.0 \times 10^{9} M_{\odot}\right.$ when including the 2 sources that were detected with SEST). 


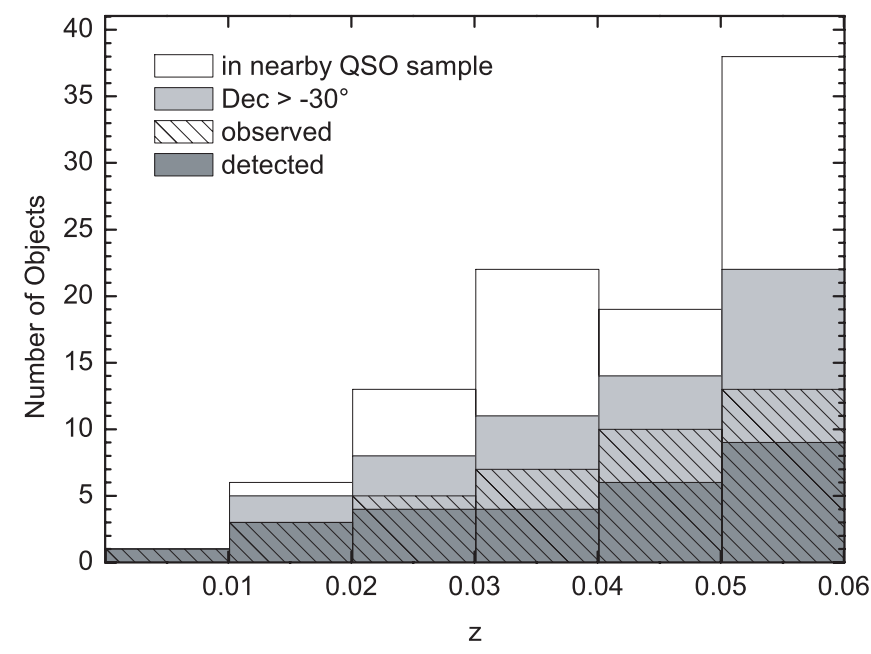

Fig. 3. The number of existing / observable with the IRAM $30 \mathrm{~m}$ telescope / observed / detected HE QSOs with increasing volume. At the distances discussed here, the frequency of occurrence is dominated by the size of the comoving volume.

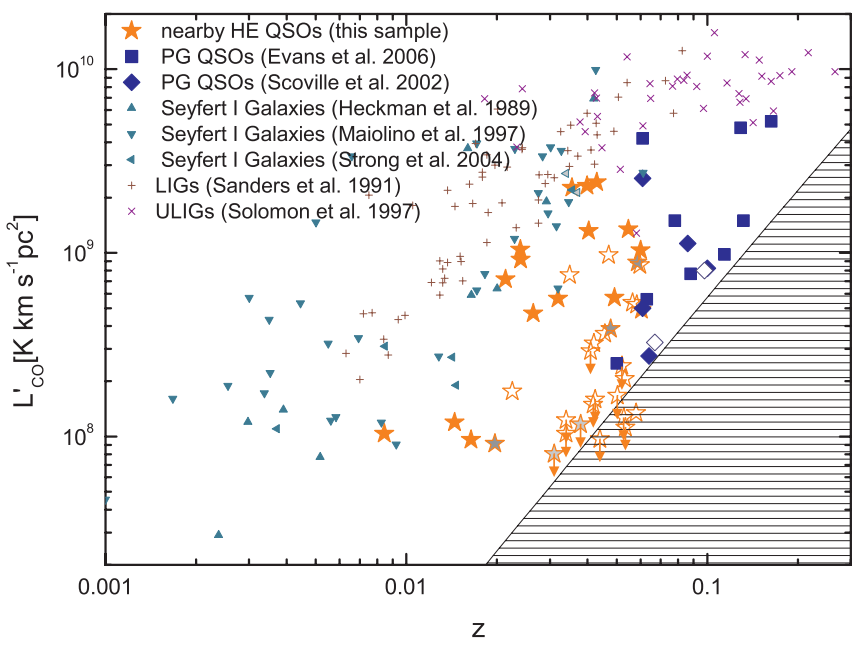

Fig. 4. CO luminosity versus redshift. For objects represented by filled shapes $L_{\mathrm{FIR}}$ is available, grey shapes indicate available upper limits for $L_{\mathrm{FIR}}$ and outlined shapes represent objects that have not been listed in the IRAS FSC. The shaded area in the bottom right corner indicates the region in which the determination of $I_{\mathrm{CO}}$ with the $30 \mathrm{~m}$ telescope falls below $0.315 \mathrm{~K} \mathrm{~km} \mathrm{~s}^{-1}$. This value corresponds to a signal of $1 \mathrm{mK}$ with a linewidth of $315 \mathrm{~km} \mathrm{~s}^{-1}$, the average width of the detected lines. (This figure is available in color in electronic form.)

\subsection{Comparison of $\mathrm{CO}$ and optical redshifts}

Millimetric CO observations are well suited for the accurate measurement of systemic velocities of active galaxies. $\mathrm{CO}$ traces the cold and extended molecular gas distribution of the total host galaxy rather than the reservoir of highly excited gas in proximity to the central source. Features of the latter are commonly used to determine the redshift of active galaxies in the visible wavelength domain. These redshifts, however, can be affected by outflows, dust obscuration or other asymmetric phenomena that result in apparent velocities deviating from the actual systemic velocity.

For nearby objects like the sample discussed in this paper, the redshifts determined in the course of the HES followup spectroscopy program are mainly based on the narrow [O III] $\lambda 5007$ emission line (Reimers et al. 1996). Boroson (2005) shows that the [O III] line is blue-shifted relative to the systemic velocity in

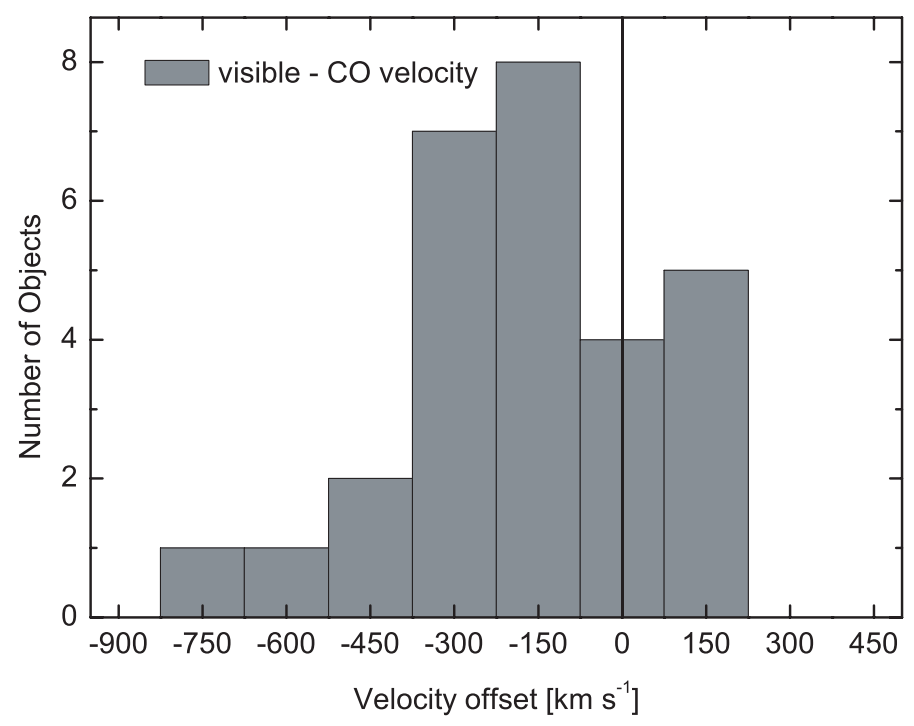

Fig. 5. The distribution of the differences between the CO velocity and the velocity derived from restframe visible spectroscopy. The systematic blueshift of the spectroscopic features determined in the visible is an indication for outflows or partial dust obscuration in the vicinity of the active nucleus.

Table 3. Mean $\mathrm{CO}(1-0)$ line widths and standard deviations of the mean for different line shapes and different absolute blue magnitudes. The numbers in parentheses represent the number of objects that fall into the corresponding category.

\begin{tabular}{lccc}
\hline \hline & $\wedge$ & $\sqcap$ & $\wedge+\Pi$ \\
\hline$M\left(B_{J}\right)>-20$ & $132 \pm 13(3)$ & $301 \pm 34(11)$ & $265 \pm 33(14)$ \\
$M\left(B_{J}\right)<-20$ & $200 \pm 28(4)$ & $426 \pm 49(11)$ & $367 \pm 45(15)$ \\
all & $171 \pm 22(7)$ & $364 \pm 32(22)$ & $317 \pm 29(29)$ \\
\hline
\end{tabular}

up to $50 \%$ of all AGN. The emission peak shift may be as large as several hundred $\mathrm{km} \mathrm{s}^{-1}$. Figure 5 shows the distribution of offsets between the velocities corresponding to the HE redshifts and the $\mathrm{CO}$ velocities measured in this study. Negative velocity differences imply blue-shifted visible emission features. On top of the nominal uncertainty of HE velocities $\left(\sim 300 \mathrm{~km} \mathrm{~s}^{-1}\right)$, the nearby low-luminosity QSOs indeed show a tendency for such a blue-shift with a mean velocity offset of $-174 \mathrm{~km} \mathrm{~s}^{-1}$.

\subsection{Blue luminosity and CO line characteristics}

Figure 6 shows the connection between absolute $B_{J}$ brightness and $\mathrm{CO}$ luminosity. The $B_{J}$ fluxes have a photometric uncertainty of $\sim 0.2 \mathrm{mag}$ and were determined by the HES group, based on their spectral plate data. Each value represents the flux within the central seeing disk, which for type I AGN at these redshifts is dominated by the nuclear contribution, with only small proportions from the host galaxy. A more detailed description is given in Wisotzki et al. (2000). The magnitudes shown here are not corrected for extinction and, therefore, systematically too faint by a mean value of $\sim 0.23 \mathrm{mag}$.

The data does not allow to distinguish between host and AGN contribution. Thus an increase of $B_{J}$ luminosity can be the result of enhanced star formation activity in the central 1-2 kpc, enhanced AGN activity or both. In any way, the increase in activity comes along with larger reservoirs of molecular gas, as can be seen in Fig. 6. Except for 3 non-detected 


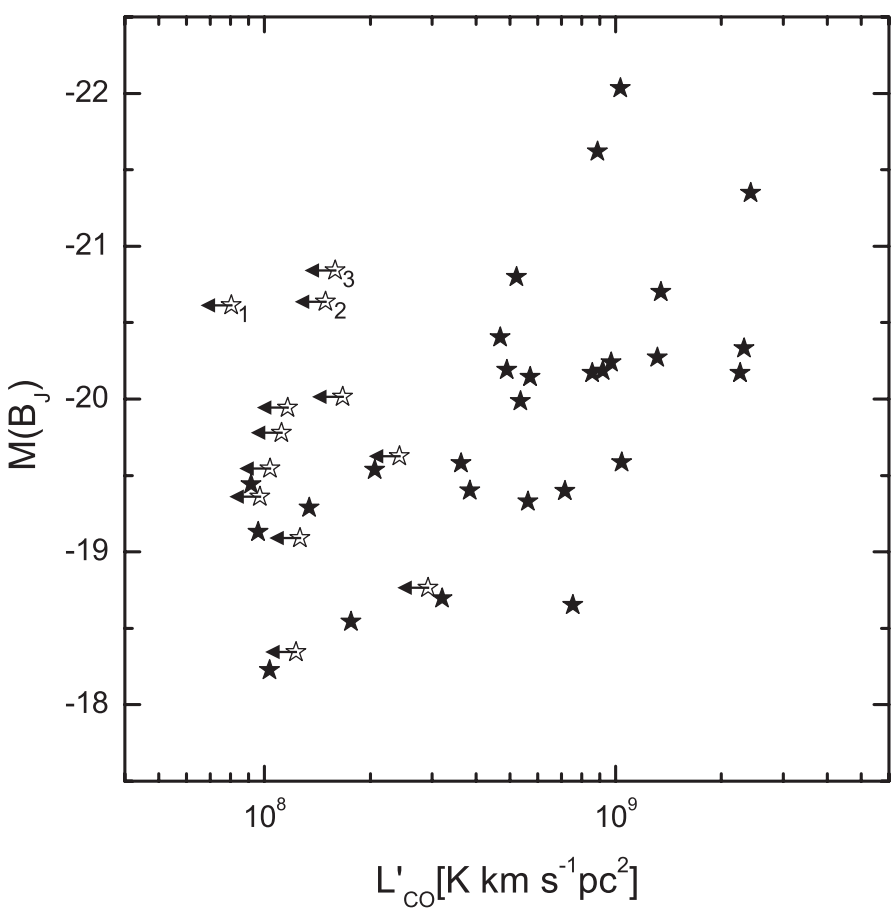

Fig. 6. Absolute $B_{J}$ magnitude versus $\mathrm{CO}$ luminosity. The magnitudes shown here represent the central seeing disk, including contributions of both AGN and star formation in the central part of each galaxy. The majority of objects showing $M\left(B_{J}\right)$ brighter than -20 mag are also CO luminous. Exceptions are (1) HE 0345+0056, (2) HE 1338-1423, and (3) HE 0203-0031.

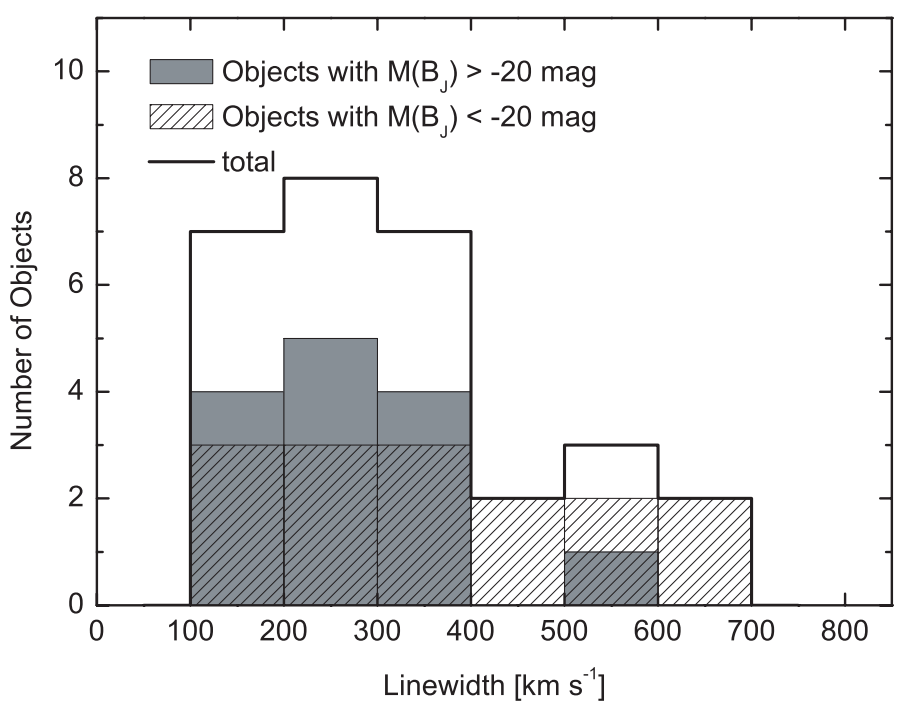

Fig. 7. Distribution of CO line widths (FWZI). Linewidths exceeding $400 \mathrm{~km} \mathrm{~s}^{-1}$ can be commonly found only in bright objects with absolute blue magnitudes $<-20$.

sources, low-luminosity QSOs with absolute central magnitudes brighter than $M\left(B_{J}\right)=-20 \mathrm{mag}$ all have $\mathrm{CO}$ luminosities of $\geq 5 \times 10^{8} \mathrm{~K} \mathrm{~km} \mathrm{~s}^{-1} \mathrm{pc}^{2}$, while the majority of fainter objects contain less molecular gas. This correlation seems to be intrinsic and not the result of a distance selection bias (with more luminous AGN being at larger distances with higher CO detection limits), as the small fraction of non-detections in the bright case shows. It remains unclear, whether the $3 B_{J}$ luminous non-detections are actually gas depleted or whether they were not detected because of an uncertain $\mathrm{CO}$ redshift. Upcoming $\mathrm{HI}$ observations, but also analyses of the morphologies in the visible/NIR will provide further information on the nature of these objects.

Not only the total CO content is higher but also the spread of the $\mathrm{CO}(1-0)$ line widths is wider for the brighter AGN hosts. Figure 7 shows the distribution of line widths in all detected galaxies. With one exception, all objects showing FWZI line widths broader than $400 \mathrm{~km} \mathrm{~s}^{-1}$ have absolute central magnitudes of $M\left(B_{J}\right) \leq-20 \mathrm{mag}$. The average line width of these bright objects is $367 \mathrm{~km} \mathrm{~s}^{-1}, \sim 100 \mathrm{~km} \mathrm{~s}^{-1}$ broader than the fainter sources (cf. Table 3 ). The average signal-to-noise ratio $(\mathrm{S} / \mathrm{N})$ of the $\mathrm{CO}(1-0)$ detections in the $B_{J}$ luminous case is only slightly (1.2 times) larger than the average $\mathrm{S} / \mathrm{N}$ in the fainter case. Therefore, a significant $\mathrm{S} / \mathrm{N}$ bias that potentially favors the identification of broader line profiles in more active objects is not present.

Such a different behavior could result from a selection effect in the optical identification process of QSO candidates. Objects with faint AGN activity may only be identified as type I AGN host when seen almost face-on, leaving the Seyfert I nucleus unobscured, while brighter AGN contributions can also be identified in objects with higher inclination. A scenario of an intrinsically broader line width distribution, however, cannot be ruled out. A separation of sources into a class of triangular shaped and a second class of box shaped or double horn line profiles resulted in similar counts for each class (cf. Tables 2 and 3 ) in both the faint and the bright cases. The triangular shaped lines are indicative for a face-on view onto a turbulent gas distribution without a dominating disk component in the line-of-sight direction. The second class represents objects with rotating $\mathrm{CO}$ emission disks or rings at a certain inclination with respect to the line-of-sight. In several low-signal cases the assignment of a class was not obvious. Nevertheless, an aforementioned selection effect that favors low-inclination views on faint AGN candidates should yield a higher fraction of triangular shaped line profiles, which is not the case. Noting the caveat of small number statistics, we would like to point out the difference in the mean line widths of triangular shaped line profiles. Bright objects with triangular shaped line profiles have a mean line width of $200 \mathrm{~km} \mathrm{~s}^{-1}$, whereas in the faint cases the mean line width is only $\sim 130 \mathrm{~km} \mathrm{~s}^{-1}$. This difference could be a sign of higher velocity dispersion in the molecular gas distribution of the more active objects.

Most of the line profiles do not show strong asymmetries that can be used as indication for ongoing interaction. Clear asymmetries can be seen in the two sources that are probably larger than the telescope beam (HE 0212-0059 and HE 0232-0900). In these cases pointing errors are the likely explanation for the asymmetric line profile.

\subsection{Far infrared luminosity and star formation efficiency}

The correlation between molecular gas and far infrared emission is well known and often discussed in the context of $\mathrm{CO}$ studies. $L_{\mathrm{FIR}} / L_{\mathrm{CO}}^{\prime}$ or $L_{\mathrm{FIR}} / M\left(\mathrm{H}_{2}\right)$ is commonly referred to as $\mathrm{Star}$ Formation Efficiency (SFE) indicator, assuming that $L_{\mathrm{CO}}^{\prime}$ traces the cold, gravitationally bound molecular gas reservoirs that form the birth places of young, dust enshrouded stars. However, $\mathrm{CO}$ is only an indirect measure as it actually traces the overall molecular gas content in galaxies rather than explicitly the dense cores that produce young stars (e.g., Gao \& Solomon 2004). Furthermore, the far IR emission of galaxies is a composite of an active star formation and a quiescent cirrus-like component (Helou 1986). In the case of AGN hosts, AGN driven dust heating may also contribute significantly to the FIR luminosity. 
Table 4. Far infrared properties of the IRAS detected sources in the subsample. The 60 and $100 \mu \mathrm{m}$ IRAS flux densities are taken from the IRAS Faint Source Catalog (Moshir \& et al. 1990), or in few cases from Rice et al. (1988), Sanders et al. (1989), or Sanders et al. (2003).

\begin{tabular}{ccrr}
\hline Obj. & $\begin{array}{c}F_{60 \mu \mathrm{m}} \\
{[\mathrm{Jy}]}\end{array}$ & $\begin{array}{c}F_{100 \mu \mathrm{m}} \\
{[\mathrm{Jy}]}\end{array}$ & $\begin{array}{r}L_{\mathrm{FIR}} \\
{\left[10^{10} L_{\odot}\right]}\end{array}$ \\
\hline HE 0045-2145 & 3.6 & 5.3 & 4.2 \\
HE 0108-4743 & 1.0 & 2.2 & 1.7 \\
HE 0119-0118 & 1.5 & 1.8 & 10.8 \\
HE 0150-0344 & 0.5 & $<1.7$ & $<4.4$ \\
HE 0212-0059 & 0.5 & 1.5 & 1.2 \\
HE 0224-2834 & 0.4 & 0.4 & 3.3 \\
HE 0227-0913 & 0.4 & 0.9 & 0.3 \\
HE 0232-0900 & 1.4 & 1.9 & 6.5 \\
HE 0253-1641 & 0.7 & 0.8 & 1.6 \\
HE 0345+0056 & 0.5 & $<3.2$ & $<2.7$ \\
HE 0412-0803 & 0.6 & $<1.4$ & $<2.8$ \\
HE 0433-1028 & 2.7 & 4.2 & 9.1 \\
HE 0949-0122 & 1.3 & $<2.4$ & $<1.5$ \\
HE 1011-0403 & 0.2 & $<0.3$ & $<1.5$ \\
HE 1017-0305 & 0.5 & 0.6 & 2.8 \\
HE 1029-1831 & 2.6 & 3.7 & 10.8 \\
HE 1108-2813 & 3.1 & 4.2 & 6.5 \\
HE 1126-0407 & 0.7 & 1.2 & 0.8 \\
HE 1237-0504 & 3.1 & 6.0 & 0.5 \\
HE 1248-1356 & 0.8 & 1.3 & 3.2 \\
HE 2211-3903 & 0.8 & 1.1 &
\end{tabular}

Many samples are based on IR selection criteria and, therefore, represent only the IR and CO bright tail of the luminosity distribution. So do several studies of the molecular gas content of Seyfert galaxies (e.g. Heckman et al. 1989; Papadopoulos \& Seaquist 1998; Strong et al. 2004) or the study of PG QSO host galaxies by Evans et al. (2001, 2006). For the sample discussed in this paper an IR selection criterion was not introduced. Only $\sim 50 \%$ of the nearby HE QSOs sample are listed in IRAS catalogs. The subsample discussed here contains the same fraction of IRAS sources (cf. Table 4) - an IR bias was carefully avoided. $65 \%$ of the detected sources are listed in IRAS catalogs, while only 2 of the 12 non-detections are detected by IRAS. Only HE 2302-0857 is located in a field that was not scanned by IRAS.

Many of the IRAS detected sources only show $60 \mu \mathrm{m}$ and $100 \mu \mathrm{m}$ flux densities and several of them are close to the IRAS detection limit. Due to the limited data at hand, the following discussion will focus on $L_{\mathrm{FIR}}$. It is based on the $60 \mu \mathrm{m}$ and $100 \mu \mathrm{m}$ IRAS flux densities and represents the luminosity between $43 \mu \mathrm{m}$ and $123 \mu \mathrm{m}$. Due to this bandwidth limit, warmer components peaking at shorter wavelengths, especially potential AGN heated dust components remain less considered in $L_{\mathrm{FIR}}$. In the context of LIGs/ULIGs or nearby galaxies, $L_{\mathrm{IR}}$ is often used instead of $L_{\mathrm{FIR}}$. $L_{\mathrm{IR}}$ also includes the $12 \mu \mathrm{m}$ and $25 \mu \mathrm{m}$ IRAS bands and represents the total IR luminosity ranging from 8 to $1000 \mu \mathrm{m}$. For the definition of $L_{\mathrm{FIR}}$ and $L_{\mathrm{IR}} \mathrm{cf}$. Sanders \& Mirabel (1996).

Figure 8 shows the distribution of the IRAS detected sources in the $L_{\mathrm{CO}}^{\prime}-L_{\mathrm{FIR}}$ diagram, together with the $\mathrm{CO}$ data of a volume limited sample of nearby spiral galaxies (Sage 1993), Seyfert I galaxies (Heckman et al. 1989; Maiolino et al. 1997; Strong et al. 2004), luminous (LIGs, Sanders et al. 1991) and ultraluminous infrared galaxies (ULIGs, Solomon et al. 1997), and PG QSOs (Scoville et al. 2003; Evans et al. 2006). Also shown are linear regression fits (in log-log space) to normal and to luminous IR galaxy samples as well as to the PG QSO hosts.

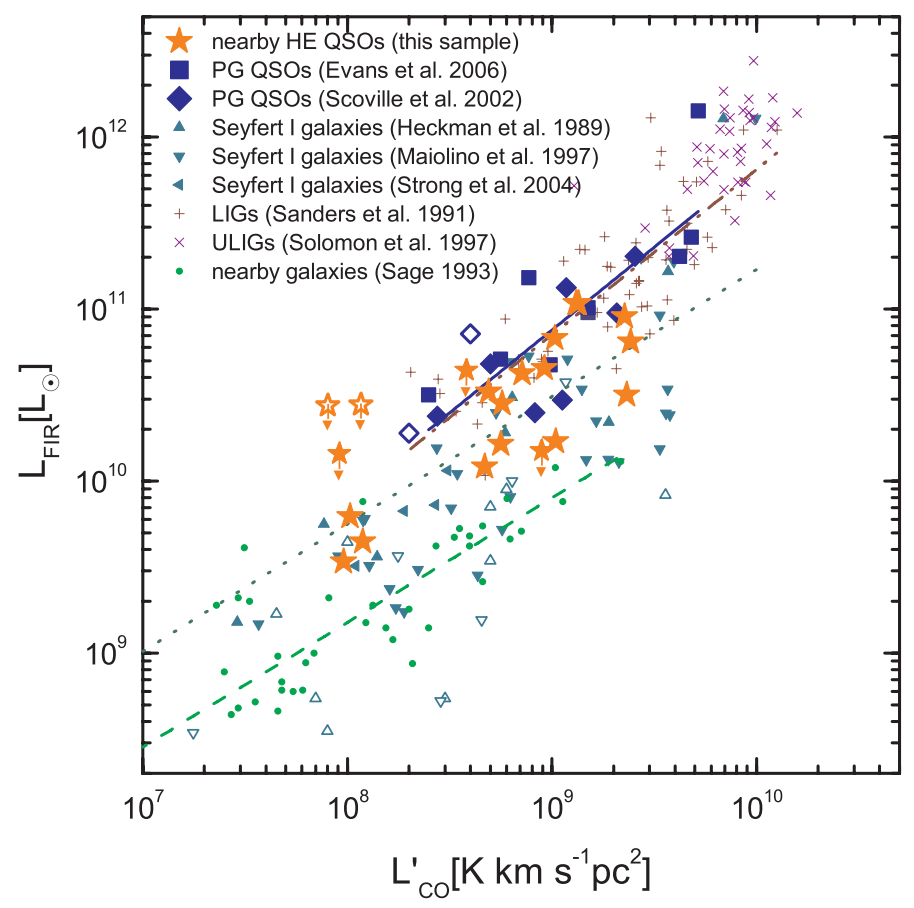

Fig. 8. FIR luminosity as a function of CO luminosity for the nearby QSO sample and various other Seyfert I, PG QSO host, luminous infrared galaxies and normal galaxies. The solid line represents a power law fit to the PG QSO hosts, the dashed line the distance limited sample of normal galaxies of Sage (1993), the dotted line a flux-limited sample of normal spiral galaxies (Solomon \& Sage 1988), and the dasheddotted line represents a power-law fit to the LIG sample of Sanders et al. (1991). Outlined symbols represent upper $L_{\mathrm{CO}}^{\prime}$ limits. (This figure is available in color in electronic form.)

The distribution of type I AGN hosts in the plot indicates the presence of two populations with differing power-laws: one population that follows the power-law of normal spiral galaxies and a second population that has $L_{\mathrm{FIR}} / L_{\mathrm{CO}}^{\prime}$ properties very similar to LIGs.

The studies of Solomon \& Sage (1988) and Sage (1993) are used to quantify the power law for normal galaxies: A fit to the distance limited sample of normal galaxies (excluding the upper limits considered in Sage 1993) resulted in

$L_{\mathrm{FIR}} / 10^{9}=8 \cdot\left[L_{\mathrm{CO}}^{\prime} / 10^{9}\right]^{0.72( \pm 0.05)}$,

while a fit to a FIR-selected sample of isolated spiral galaxies or spiral galaxies that do not show signs of interaction (Solomon \& Sage 1988) yielded

$L_{\mathrm{FIR}} / 10^{9}=31 \cdot\left[L_{\mathrm{CO}}^{\prime} / 10^{9}\right]^{0.74( \pm 0.07)}$.

Due to the FIR selection criterion, the latter sample is biased towards more active star formation with respect to average spiral galaxies. The bulk of local Seyfert I galaxies follows the trend described by these two fits, with a somewhat higher $L_{\mathrm{FIR}} / L_{\mathrm{CO}}^{\prime}$ ratio for any given $L_{\mathrm{CO}}^{\prime}$ than the Sage (1993) sample.

The power law fit to the PG QSO hosts, on the other hand, produces a slope of about unity:

$L_{\mathrm{FIR}} / 10^{9}=75 \cdot\left[L_{\mathrm{CO}}^{\prime} / 10^{9}\right]^{0.96( \pm 0.19)}$

and is almost identical to the fit to the LIG data:

$L_{\mathrm{FIR}} / 10^{9}=71 \cdot\left[L_{\mathrm{CO}}^{\prime} / 10^{9}\right]^{0.96( \pm 0.09)}$.

This common trend may be indicative for similar star formation activity in LIGs and luminous QSO hosts. 
As for the low-luminosity QSO sample, Fig. 8 supports the picture of the sample being a link between the local Seyfert I population and luminous QSO host galaxies. The sample members seem to follow both trends, most of them can be found in a transition region between the two populations.

\subsection{Opaque CO and FIR emission in (U)LIGs and QSO host galaxies?}

In the following we will summarize the various published results on the $L_{\mathrm{CO}}^{\prime}-L_{\mathrm{FIR}}$ relation in normal and infrared luminous galaxies together with the findings on the nature of the emission regions. We combine different pieces of information to a simple qualitative model that explains geometrically the separation of type I AGN hosts into two populations in the $L_{\mathrm{FIR}}$ range between $10^{9} L_{\odot}$ and $5 \times 10^{11} L_{\odot}$.

As discussed in Sect. 5.4, normal, undisturbed galaxies show a nonlinear $L_{\mathrm{CO}}^{\prime}-L_{\mathrm{FIR}}$ relation with a slope $<1$. For interacting galaxies (Solomon \& Sage 1988) and LIGs (Sanders et al. 1991) the slope is approaching unity. In this case a linear relation between $\mathrm{CO}$ and FIR emission can be assumed. For ULIGs Solomon et al. (1997) also find a linear relation between FIR and $\mathrm{CO}$ emission, with a higher $L_{\mathrm{FIR}} / L_{\mathrm{CO}}$ ratio than in normal galaxies and LIGs. The increase of the $L_{\mathrm{FIR}} / L_{\mathrm{CO}}$ ratio towards ULIGs is well known (e.g., Sanders et al. 1986; Sanders \& Mirabel 1996; Gao \& Solomon 2004). It extends towards the even more luminous SMGs (Greve et al. 2005). Gao \& Solomon (2004) point out, that a linear relation between $\mathrm{CO}$ and FIR luminosity remains valid only over two orders of magnitude in the lower $L_{\text {FIR }}$ range (while a corresponding $\mathrm{HCN}$ - FIR relation extends over three orders of magnitude and includes the ULIG domain). Any fit to the CO luminosity over a wider range naturally yields a slope $>1$. Gao \& Solomon state a slope of $1.25 \pm 0.08$ for $L_{\mathrm{FIR}}$ between $10^{9.5} L_{\odot}$ and $10^{12.5} L_{\odot}$. Yao et al. (2003) even obtain a slope of 1.7 for $L_{\mathrm{FIR}}$ between $10^{9} L_{\odot}$ and $10^{12} L_{\odot}{ }^{2}$.

In interferometric CO data on nearby ULIGs Downes \& Solomon (1998) show that most of the emission originates from rotating circumnuclear disks or rings. The disk gas forms a continuous medium rather than discrete virialized clouds. The linearity between FIR and CO emission in ULIGs and the dense environment resulting from the massive concentration of molecular gas suggest thermal excitation and optically thick emission. This is proposed by the same authors in an earlier paper (Solomon et al. 1997) but partially revised in Downes \& Solomon (1998): According to their model fits the CO line emission is only moderately opaque and the $\mathrm{CO}(2-1) / \mathrm{CO}(1-0)$ ratio indicates subthermal excitation.

If a connection between ULIGs and QSOs exists, as suggested in the evolutionary model, what does this imply for the molecular gas content in the evolved QSO phase of these objects? The model assumes a dispersion of the majority of gas and interstellar dust to clear the view onto the formerly dust enshrouded AGN. Indeed, low-z PG QSOs and nearby HE QSOs show $\mathrm{CO}$ and FIR luminosities and star formation efficiencies that are lower than it is the case for ULIGs in the same comoving volume. Nevertheless, the majority of nearby lowluminosity QSOs is associated with large reservoirs of molecular gas (Sect. 5.1) and presumably also with ongoing star formation, as it is indicated by their $L_{\mathrm{CO}}^{\prime}-L_{\mathrm{FIR}}$ distribution (Fig. 8).

2 Their small projected beam size, however, may not cover the full $\mathrm{CO}$ content especially in the nearby, less infrared luminous galaxies. Thus, the slope may appear steeper than it actually is.
This might imply that the density of the formerly AGN obscuring medium in the central region is significantly reduced.

But how does the evolution affect the distribution of molecular gas? A confinement of the molecular gas to a compact region is still indicated also in high redshift QSOs (Riechers et al. 2006 , in ground-state transition observations at $z \gtrsim 4)$. Spatially resolved data on QSOs in the local universe, however, is hardly available. For Mrk 231 Downes \& Solomon (1998) report on an inner and an outer face-on disk within a radius of $1.15 \mathrm{kpc}$. Staguhn et al. (2004) present a ringlike structure with a radius of $\sim 1.2 \mathrm{kpc}$ in the circumnuclear molecular gas distribution of I Zw 1. For the low-luminosity QSO HE 1029-1831 Krips et al. (2007) estimates a size of $6 \pm 2 \mathrm{kpc}$ for the CO source that is aligned with the optical bar. The interferometric maps of the low- $z$ PG QSOs by Evans et al. (2001) and Scoville et al. (2003) do not permit to draw conclusions on the compactness of the emission.

The coincidence with the LIG population in the $\log L_{\mathrm{CO}}^{\prime}$ $\log L_{\text {FIR }}$ diagram with a power-law index 1 can be taken as indicator for a confinement of remaining $\mathrm{H}_{2}$ content to a small and dense region. Based on this assumption, we would like to revisit the idea of both bands being optically thick. If this is the case, the two different inclinations in the $\log L_{\mathrm{CO}}^{\prime}-\log L_{\mathrm{FIR}}$ diagram may have a simple geometrical explanation: For normal galaxies, the CO distribution is less confined. The totality of individual gas clouds with varying line-of-sight velocities contributes to the spectrum. In this case, it is appropriate to use the velocityintegrated $\mathrm{CO}$ line emission as tracer for the total molecular gas content of the galaxy. The FIR luminosity, on the other hand, is dominated by reradiated dust emission, which originates from the densest regions in the ISM, the seeds of star formation. If the column densities in these compact regions result in $\tau>1$ at the FIR bands, only the radiating surface contributes to the detectable FIR flux density. Let us assume a coarse, linear relation between the molecular gas content and the number of star formation seeds in a 3-dimensional gas distribution. Any surface of optically thick emission then scales with power of $\frac{2}{3}$ of the overall volume.

If $L_{\mathrm{CO}}^{\prime}$ can be used as measure for the total molecular gas content, $L_{\mathrm{FIR}}$ should scale with $L_{\mathrm{CO}}^{\prime 0.67}$ and produce the corresponding inclination in the log-log plot. Once the density of the ISM reaches a threshold at which the $\mathrm{CO}$ emitting region (which may consist of a continuous medium) becomes opaque, both the $\mathrm{CO}$ and the FIR emitting surface scale linearly with the overall molecular gas and dust content. Under these circumstances the slope in the log-log plot is close to unity. An even higher density of star formation seeds in the ISM results in a larger surface filling factor within the galaxy, allowing for a higher $L_{\mathrm{FIR}} / L_{\mathrm{CO}}^{\prime}$ ratio for each given $\mathrm{CO}$ luminosity. This effect causes the dispersion perpendicular to the power-law fit. As shown in the Appendix the different power-law fits for the individual source classes can be parameterized via the fractional source filling factor $a$ of the material that participates in intense star formation or the source type $f$ (QSO hosts and LIGs/ULIGs or normal galaxies).

The LIG/QSO scenario and the normal galaxy scenario describe the two extreme cases with power-laws 1 and 0.67 as upper and lower limit. These extreme cases seem to correspond well to the two populations described above. Furthermore, these scenarios do not exclude a transition region, in which the dense central region of a galaxy is optically thick for both bands while for the outer regions the full volume contributes to the detected $\mathrm{CO}$ emission. 


\section{Summary}

This paper reports on ${ }^{12} \mathrm{CO}$ observations of 41 nearby lowluminosity QSOs. Thirty-nine of them form a subsample that is not biased towards FIR emission. The results of this study can be summarized as follows:

1. $70 \%$ of the subsample have been detected in the ${ }^{12} \mathrm{CO}(1-0)$ transition. With a mean $\mathrm{H}_{2}$ mass of $2.8 \times 10^{9} M_{\odot}$ (assuming $\left.\alpha=4 M_{\odot}\left(\mathrm{K} \mathrm{km} \mathrm{s}^{-1} \mathrm{pc}^{2}\right)^{-1}\right)$ the majority of low-luminosity QSO hosts is rich in molecular gas. This confirms previous results on PG QSOs by Scoville et al. (2003).

2. For the majority of detected objects, the redshift based on visible features is blueshifted with respect to the $\mathrm{CO}$ line centroid - probably as a result of asymmetric phenomena in the vicinity of the nucleus, the emission region of the visible features.

3. The absolute $B_{J}$ magnitude of the central seeing disk of the low-luminosity QSOs is used as an indicator for the total activity of AGN and circumnuclear starburst. Objects with $M\left(B_{J}\right)<-20$ mag not only show higher CO luminosities but also a wider spread in the distribution of linewidths than the fainter objects.

4. The connection between CO and FIR properties is discussed in the context of normal galaxies, local Seyfert I objects, LIGs, ULIGs and PG QSOs. Two populations with different power-laws in the $L_{\mathrm{CO}}^{\prime}-L_{\mathrm{FIR}}$ plot are identified in the range between $L_{\mathrm{FIR}}=10^{9} L_{\odot}$ and $L_{\mathrm{FIR}}=5 \times 10^{11} L_{\odot}$. The first population shows a power-law index of $\sim 0.7$ and contains normal, non- or weakly interacting galaxies and the bulk of Seyfert I objects. The second population with a powerlaw index of $\sim 1$ contains LIGs and the PG QSOs. The lowluminosity QSO sample seems to consist of objects of both populations and objects from a transition region.

5. For Objects of the second population, i.e. also for galaxies hosting brighter QSOs, the linear relation between FIR and $\mathrm{CO}$ luminosity can be taken as indication for a concentration of the molecular gas in compact regions, similar to the case of ULIGs.

6. The idea of thermalized, optically thick FIR and CO emission, previously suggested in the context of ULIGs, is revisited. The two different slopes in the $L_{\mathrm{CO}}^{\prime}-L_{\mathrm{FIR}}$ plot are discussed as a potential result of different optical properties of the ISM in the two populations.

Acknowledgements. T.B. would like to thank the staff of the IRAM $30 \mathrm{~m}$ telescope for their kind support. We thank the anonymous referee for helpful comments. Part of this work was supported by the Deutsche Forschungsgemeinschaft (DFG), project number: SFB 494.

\section{Appendix A: Descriptive star formation model}

Acknowledging the opaqueness of a dense, potentially continuous, medium and the importance of the contribution of individual star formation regions to the overall emission leads to the consequence that the sources are characterized by the surface filling factor $a$ of the star forming molecular gas in the host and a quantity $\beta$ that describes the $L_{\mathrm{FIR}}$ and $L_{\mathrm{CO}}^{\prime}$ contributions of the emitting regions (see below). Here $L_{\mathrm{CO}}^{\prime}$ alone is not necessarily an adequate measure of the total molecular gas content in dense environments (as outlined in Sect. 5.4). Only if the standard assumptions are valid (virialized, i.e. gravitationally bound giant molecular clouds with different line-of-sight velocities), $I_{\mathrm{CO}}$ traces molecular gas in both the LIG/ULIG/PG QSO case and the normal galaxy case.

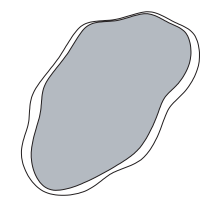

$\mathrm{a} \sim 1.0$

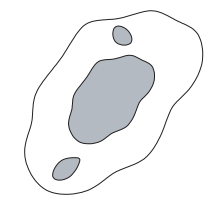

$1.0<\mathrm{a}<0.1$

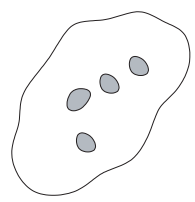

a 0.1
Fig. A.1. Examples of molecular gas distributions with different fractions of molecular gas taking part in active star formation (dark areas). We show an inclined host and the outer continuous line includes the majority of the molecular gas in the system.

The two extreme populations (LIG/ULIGs/PG QSOs and normal galaxies) in Fig. 8 can then be described as follows. For PG QSO hosts and LIGs CO and FIR luminosity are directly proportional:

$\log \left(L_{\mathrm{CO}}^{\prime}\right) \propto \log \left(L_{\mathrm{FIR}}\right)$.

For normal galaxies, additional quiescent molecular gas reservoirs contribute to the object's total CO luminosity but do not, or at least not as efficiently, produce dust emission in the FIR. The additional contribution grows non-linear with increasing total molecular mass. The portion of CO-luminosity per FIRluminosity is higher by a factor $(1+\beta)$, with $\beta>0$ :

$\log \left(L_{\mathrm{CO}}^{\prime}\right) \propto[1+\beta] \log \left(L_{\mathrm{FIR}}\right) \sim 1.4 \log \left(L_{\mathrm{FIR}}\right)$.

As a first step we assume in the following a smooth transition between the host properties of these different classes, which can be parameterized by the value $f$ with $0 \leq f \leq 1$. $f=1$ represents the case of QSO hosts and LIGs/ULIGs, while $f=0$ represents the case of normal galaxies. Furthermore, a direct proportionality between the CO and FIR luminosity in the $f=1$ case and in the star forming portions of normal galaxies is assumed. We also assume that in the $f=1$ case the molecular gas of a dominant part of the host participates in the star formation process. In normal galaxies only a fraction $a-$ for which $\log \left(L_{\mathrm{CO}}^{\prime}\right) \propto \log \left(L_{\mathrm{FIR}}\right)$ is valid - participates in that process. The quantity $a$ can be identified as the surface filling factor of the star forming molecular gas in the host with $0<a<1$ (cf. Fig. A.1). The remaining part gives rise to $\beta \log \left(L_{\mathrm{FIR}}\right)$ as an additional portion of CO-luminosity. With a parameter $C_{0}$ we can then write:

$$
\begin{aligned}
\log \left(L_{\mathrm{CO}}^{\prime}\right) \cong & f \log \left(L_{\mathrm{FIR}}\right) \\
& +(1-f)\left[\log \left(a L_{\mathrm{FIR}}\right)+\beta \log \left((1-a) L_{\mathrm{FIR}}\right)\right]+C_{0} \\
= & {[1+(1-f) \beta] \log \left(L_{\mathrm{FIR}}\right) } \\
& +(1-f)[\log (a)+\beta \log (1-a)]+C_{0}
\end{aligned}
$$

In Fig. A. 2 we show as a second step the interdependency of $a$ and $f$ as they result in suitable power-law fits to the extreme and intermediate source classes in Fig. 8. We find that both quantities are linked via

$\log (a) \cong 3(f-1)$.

Within this model the two extreme source populations are represented by the following cases:

Case I: for $f=1$, i.e. for QSO hosts and LIGs/ULIGs with $a \simeq 1$ follows:

$\log \left(L_{\mathrm{CO}}^{\prime}\right) \cong \log \left(L_{\mathrm{FIR}}\right)+C_{0}$

With a value of $C_{0} \sim-2$, as suggested by Fig. 8, this corresponds to the heuristically derived expressions for LIGs/ULIGs and PG 


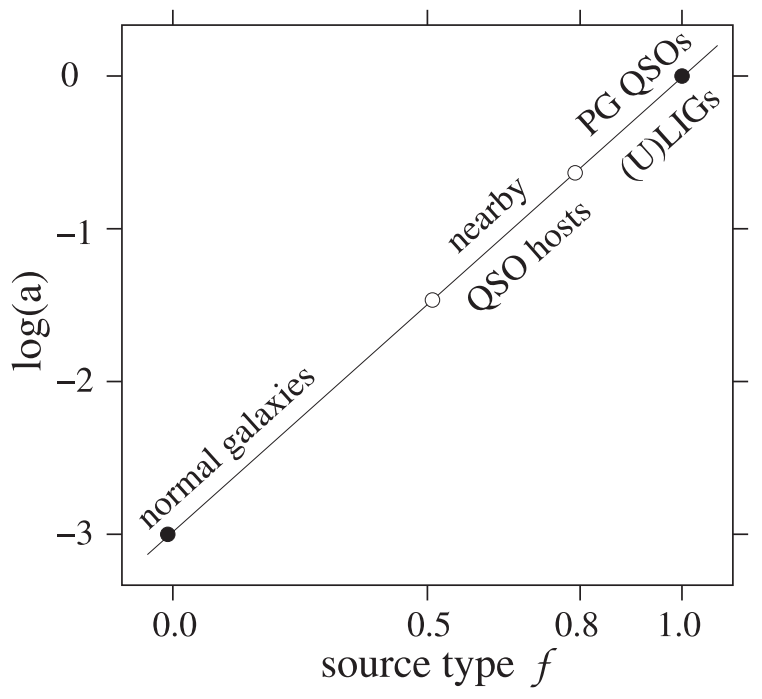

Fig. A.2. Logarithm of the surface filling factor $a$ of star-forming gas as a function of source type $f$ as described in the text. The black filled circles mark the fits to the LIG/ULIG and the normal galaxies, respectively. The white filled circle indicate the region with which the nearby low-luminosity QSO hosts (discussed in this paper) are located.

QSOs (Sanders et al. 1991; Solomon et al. 1997; Scoville et al. 2003; Evans et al. 2006).

Case II: For normal galaxies with $f=0$, i.e. $0<a \ll 1$, and $\beta \sim 0.4$ the model yields:

$\log \left(L_{\mathrm{CO}}^{\prime}\right) \cong 1.4 \log \left(L_{\mathrm{FIR}}\right)-5.0$.

This corresponds to the heuristically derived expressions for samples of distance limited or non-interacting, isolated FIRselected galaxies (Solomon \& Sage 1988; Sage 1993, cf. Sect. 5.4). We see that the formalism described above naturally results in the heuristic relations for the extreme source populations as presented in Sect. 5.4 and shown in Fig. 8. It also allows us to describe the mean properties of nearby low-luminosity QSO hosts discussed in this paper as an intermediate population. The intermediate case of the nearby low-luminosity QSO hosts, for which $0.5 \lesssim f \lesssim 0.8$ or $\log a \sim-1$, can then be approximated by

$\log \left(L_{\mathrm{CO}}^{\prime}\right) \cong(1.1 \pm 0.05) \log \left(L_{\mathrm{FIR}}\right)-(2.5 \pm 0.3)$

or

$L_{\mathrm{FIR}} / 10^{9} \cong(50 \pm 20) \cdot\left[L_{\mathrm{CO}}^{\prime} / 10^{9}\right]^{0.91( \pm 0.04)}$.

Overall the model corresponds to a picture in which all hosts are similar in the amount of molecular gas (i.e. about $10 \%$ of $\sim 10^{12} M_{\odot}$ of the total host mass) but different in the fraction of strong star forming regions. It also shows that the surface filling factor $a$ and the value $\beta$ that is linked to the FIR/CO emission properties of the source components are suitable parameters to describe a broad range of host galaxies.

\section{References}

Barthel, P. D. 2006, A\&A, 458, 107

Bertram, T., Eckart, A., Krips, M., et al. 2006, New Astron. Rev., 50, 712 Boroson, T. 2005, AJ, 130, 381

Curran, S. J., Aalto, S., \& Booth, R. S. 2000, A\&AS, 141, 193

Downes, D. \& Solomon, P. M. 1998, ApJ, 507, 615

Dunlop, J. S., McLure, R. J., Kukula, M. J., et al. 2003, MNRAS, 340, 1095

Evans, A. S., Frayer, D. T., Surace, J. A., \& Sanders, D. B. 2001, AJ, 121, 3285

Evans, A. S., Solomon, P. M., Tacconi, L. J., Vavilkin, T., \& Downes, D. 2006, AJ, 132, 2398

Fischer, S., Iserlohe, C., Zuther, J., et al. 2006, A\&A, 452, 827

Gao, Y. \& Solomon, P. M. 2004, ApJ, 606, 271

Greve, T. R., Bertoldi, F., Smail, I., et al. 2005, MNRAS, 359, 1165

Heckman, T. M., Blitz, L., Wilson, A. S., Armus, L., \& Miley, G. K. 1989, ApJ, 342,735

Helou, G. 1986, ApJ, 311, L33

Horellou, C., Casoli, F., Combes, F., \& Dupraz, C. 1995, A\&A, 298, 743

Israel, F. P. 1997, A\&A, 328, 471

Köhler, T., Groote, D., Reimers, D., \& Wisotzki, L. 1997, A\&A, 325, 502

Krips, M., Eckart, A., Neri, R., et al. 2007, A\&A, 464, 187

Lawrence, A., Rowan-Robinson, M., Ellis, R. S., et al. 1999, MNRAS, 308, 897

Maiolino, R., Ruiz, M., Rieke, G. H., \& Papadopoulos, P. 1997, ApJ, 485, 552

Moshir, M. \& et al. 1990, in IRAS Faint Source Catalogue, version 2.0, 0

Papadopoulos, P. P. \& Seaquist, E. R. 1998, ApJ, 492, 521

Reimers, D., Köhler, T., \& Wisotzki, L. 1996, A\&AS, 115, 235

Rice, W., Lonsdale, C. J., Soifer, B. T., et al. 1988, ApJS, 68, 91

Riechers, D. A., Walter, F., Carilli, C. L., et al. 2006, ApJ, 650, 604

Sage, L. J. 1993, A\&A, 272, 123

Sanders, D. B., Mazzarella, J. M., Kim, D.-C., Surace, J. A., \& Soifer, B. T. 2003, AJ, 126, 1607

Sanders, D. B. \& Mirabel, I. F. 1996, ARA\&A, 34, 749

Sanders, D. B., Phinney, E. S., Neugebauer, G., Soifer, B. T., \& Matthews, K. 1989, ApJ, 347, 29

Sanders, D. B., Scoville, N. Z., \& Soifer, B. T. 1991, ApJ, 370, 158

Sanders, D. B., Scoville, N. Z., Young, J. S., et al. 1986, ApJ, 305, L45

Sanders, D. B., Soifer, B. T., Elias, J. H., et al. 1988, ApJ, 325, 74

Schmidt, M. \& Green, R. F. 1983, ApJ, 269, 352

Scoville, N. Z., Frayer, D. T., Schinnerer, E., \& Christopher, M. 2003, ApJ, 585, L105

Scoville, N. Z., Sargent, A. I., Sanders, D. B., \& Soifer, B. T. 1991, ApJ, 366, L5

Solomon, P. M. \& Barrett, J. W. 1991, in Dynamics of Galaxies and Their Molecular Cloud Distributions, ed. F. Combes \& F. Casoli, IAU Symp. 146, 235

Solomon, P. M., Downes, D., \& Radford, S. J. E. 1992a, ApJ, 398, L29

Solomon, P. M., Downes, D., Radford, S. J. E., \& Barrett, J. W. 1997, ApJ, 478, 144

Solomon, P. M., Radford, S. J. E., \& Downes, D. 1992b, Nature, 356, 318

Solomon, P. M. \& Sage, L. J. 1988, ApJ, 334, 613

Staguhn, J. G., Schinnerer, E., Eckart, A., \& Scharwächter, J. 2004, ApJ, 609, 85

Strong, M., Pedlar, A., Aalto, S., et al. 2004, MNRAS, 353, 1151

Tacconi, L. J., Neri, R., Chapman, S. C., et al. 2006, ApJ, 640, 228

Véron-Cetty, M.-P. \& Véron, P. 1996, A Catalogue of quasars and active nuclei (ESO Scientific Report, Garching: European Southern Observatory (ESO), 7th ed.)

Vila-Vilaró, B., Taniguchi, Y., \& Nakai, N. 1998, AJ, 116, 1553

Wisotzki, L., Christlieb, N., Bade, N., et al. 2000, A\&A, 358, 77

Yao, L., Seaquist, E. R., Kuno, N., \& Dunne, L. 2003, ApJ, 588, 771

Young, J. S., Xie, S., Tacconi, L., et al. 1995, ApJS, 98, 219 